\title{
Fused Filament Fabrication Three-Dimensional Printing Multi-Functional of Polylactic Acid/Carbon Black Nanocomposites
}

\author{
Nectarios Vidakis ${ }^{1}$, Markos Petousis ${ }^{1, *}$ [, Emmanuel Velidakis ${ }^{1}{ }^{\circledR}$, Nikolaos Mountakis ${ }^{1}$, \\ Peder Erik Fischer-Griffiths ${ }^{2}$, Sotirios Grammatikos ${ }^{2}{ }^{-}$and Lazaros Tzounis ${ }^{3}$ \\ 1 Mechanical Engineering Department, Hellenic Mediterranean University, Estavromenos, \\ 71004 Heraklion, Greece; vidakis@hmu.gr (N.V.); mvelidakis@hmu.gr (E.V.); mh90@edu.hmu.gr (N.M.) \\ 2 Group of Sustainable Composites, Department of Manufacturing and Civil Engineering, \\ Norwegian University of Science and Technology, 2815 Gjøvik, Norway; pederef@stud.ntnu.no (P.E.F.-G.); \\ sotirios.grammatikos@ntnu.no (S.G.) \\ 3 Department of Materials Science \& Engineering, University of Ioannina, 45110 Ioannina, Greece; \\ latzounis@uoi.gr \\ * Correspondence: markospetousis@hmu.gr; Tel.: +30-281-037-9227
}

check for updates

Citation: Vidakis, N.; Petousis, M.; Velidakis, E.; Mountakis, N.; Fischer-Griffiths, P.E.; Grammatikos, S.; Tzounis, L. Fused Filament Fabrication Three-Dimensional Printing Multi-Functional of Polylactic Acid/Carbon Black Nanocomposites. C 2021, 7, 52. https://doi.org/10.3390/c7030052

Academic Editor: Gil Goncalves

Received: 23 June 2021

Accepted: 15 July 2021

Published: 17 July 2021

Publisher's Note: MDPI stays neutral with regard to jurisdictional claims in published maps and institutional affiliations.

Copyright: (c) 2021 by the authors. Licensee MDPI, Basel, Switzerland. This article is an open access article distributed under the terms and conditions of the Creative Commons Attribution (CC BY) license (https:/ / creativecommons.org/licenses/by/ $4.0 /)$.

\begin{abstract}
Conductive Polymer Composites (CPCs) have recently gained an extensive scientific interest as feedstock materials in Fused Filament Fabrication (FFF) Three-dimensional (3D) printing. Polylactic Acid (PLA), widely used in FFF 3D printing, as well as its Carbon Black (CB) nanocomposites at different weight percentage (wt.\%) filler loadings (0.5, 1.0, 2.5 and $5.0 \mathrm{wt} . \%)$, were prepared via a melt mixing filament extrusion process in this study and utilized to manufacture FFF 3D printed specimens. The nanocomposites were examined for their electrical conductivity. The highest loaded 3D printed CPC (5.0 wt.\%) was tested as an electrothermal Joule heating device. Static tensile, flexural, Charpy's impact and Vickers microhardness mechanical properties were investigated for the neat and PLA/CB 3D printed nanocomposites. Dynamic Mechanical Analysis (DMA) revealed a stiffening mechanism for the PLA/CB nanocomposites. Scanning Electron Microscopy (SEM) elucidated the samples' internal and external microstructural characteristics. The PLA/CB $5.0 \mathrm{wt}$.\% nanocomposite demonstrated also antibacterial properties, when examined with a screening process, against Escherichia coli (E. coli) and Staphylococcus aureus (S. aureus). It can be envisaged that the 3D printed PLA/CB CPCs exhibited a multi-functional performance, and could open new avenues towards low-cost personalized biomedical objects with complex geometry, amongst others, i.e., surgery tools, splints, wearables, etc.
\end{abstract}

Keywords: fused filament fabrication (FFF); three-dimensional (3D) printing; conductive polymer composites (CPCs); nanocomposites; electrothermal effect; antibacterial properties

\section{Introduction}

Additive Manufacturing (AM) is in the forefront of research, receiving a continuous industrial interest for potential applications in the fields of engineered polymer composites [1], printed electronics [2], personalized health and biomedical equipment [3], civil engineering infrastructure i.e., buildings, bridges, etc. [4], amongst others. AM enables the manufacturing of components and end-use products with customizable geometries. It is an efficient technology, especially when small batches are required, which are implausible for conventional manufacturing processes that present several limitations and freedom to fabricate parts with complex shapes [5]. Three-dimensional (3D) printing, which is one of the most famous AM technologies, has recently attracted interest on both academia, as well as various industrial sectors for objects' manufacturing and/or prototyping purposes [6]. To that end, it is worth mentioning that $3 \mathrm{D}$ printing is a cutting-edge technology allowing the production of $3 \mathrm{D}$ objects with more complex geometry compared to conventionally 
machined parts [5]. Among the increasing number of 3D printing processes, the most wellknown ones are: (i) Fused Filament Fabrication (FFF), (ii) Stereo-lithography, (iii) Selective Laser Sintering (SLS), (iv) Binder Jetting, (v) Material Jetting and Drop on Demand (DOD) and (vi) Digital Light processing (DLP) [7].

FFF is the most famous, widely employed and extremely emerging 3D printing technique for applications ranging from scientific development, home 3D printing and Rapid Prototyping, up to manufacturing parts at industrial scale (especially for high performance advanced 3D components). Moreover, FFF has recently penetrated various industrial sectors and became a mature technology, replacing conventional ones, i.e., Plastic Forming (PF), Computer Numerical Control (CNC) machining, (plastic) joining and injection molding [6]. In FFF, a thermoplastic polymeric material is used as the feedstock in filament form. It is being heated above its Melting Point (Tm) and extruded with a movable nozzle in the $\mathrm{X}-\mathrm{Y}$ direction to create a 3D structure via a layer-by-layer addition manner [8]. FFF could offer thus numerous advantages and a direct solution to: (i) the acute shortage of goods, as for instance in the COVID-19 pandemic situation [9], (ii) freedom of design of 3D objects, (iii) mass customization and (iv) reduced material's consumption leading to waste minimization [10].

Great achievements have been made so far regarding the FFF 3D printing technology, as for instance: (i) the 3D printer's hardware parts i.e., the heated bed, the preheated chamber of the filament, etc., (ii) the FFF 3D printers' automation and accuracy and (iii) the extruder head (metallic materials' properties towards optimum heat flow for homogeneous heat transfer to the polymeric filament and melt properties), allowing high quality AM filamentous 3D printing, that could significantly affect the 3D printed final object "bulk" properties. Nevertheless, FFF is limited by the availability of functional thermoplastic materials, with most of the existing stock filament materials properties being investigated and reported in literature so far [11-13] and a lot of research presented for the development of new polymeric materials for AM in general [14].

Functional thermoplastic filaments can endow additional functionalities to the final 3D printed object, as for instance enhanced physicochemical properties e.g., electrical, thermal, magnetic, mechanical, melt-rheological properties (allowing better processing), etc., giving an added value to the 3D printed final end-product [15]. To that end, is of great interest to investigate the thermoplastic polymer engineering part, by tuning the filament's melt rheological properties via additives, such as viscosity modifiers, polymer blends, nanoparticulate additives, etc., resulting into higher quality 3D printed parts (extensive interlayer fusion), high production rate and production yield of the FFF printing process [16].

To date, there is an increasing demand for FFF 3D printable materials exhibiting multifunctional properties, i.e., materials with enhanced physical and mechanical properties [5]. Namely, functional thermoplastic materials as filaments i.e., electrically conductive and thermoelectric [17], magnetically active [18], capacitive and piezoresistive [19], flexible and stretchable [20], mechanically reinforced [21], antimicrobial [22], etc., have been used for FFF 3D printing, yielding multifunctional 3D objects with tunable properties. Therefore, polymer nanocomposites could offer a unique strategy towards novel filaments and by extension FFF 3D printed derived objects. Specifically, for the polymer nanocomposites, one should consider the nanoparticle filler geometry, density and surface chemistry. Additionally, the nanoscale interactions should be assessed, e.g., the well-known filler-filler interaction and the filler-matrix interactions that have profound implications on the macroscopic behavior of the $3 \mathrm{D}$ printed nanocomposite objects as well as to the $3 \mathrm{D}$ printing process [23].

Polylactic acid (PLA) is a high performance biocompatible and biodegradable thermoplastic in nature engineering polymeric material, already being widely used and several times reported in FFF 3D printing [24]. In general, polymer/carbon nanoparticulate nanocomposites have attracted a significant attention over the last decade, as lightweight and flexible electrically conductive materials $[25,26]$. The resulting Conductive Polymer 
Composites (CPCs) could find numerous applications, due to the material's conductive behavior, which can be tuned by the nanoparticle filler loading, the particle aspect ratio, surface area and surface chemistry, as well as the processing/mixing parameters. Carbon black (CB) is an allotrope of carbon, while it has been widely utilized as a nanoadditive in various polymer matrices to increase specific properties, such as fracture toughness [27], tensile strength [28], electrical [29] and thermal conductivity [30]. Polymer/CB CPCs have demonstrated sensing functionalities i.e., (i) gas or liquid chemical and/or electrochemical sensors, (ii) strain sensors for wearables or touch/pressure sensors via a piezoresistive or a piezoelectric under-lying mechanism and (iii) temperature sensors [31]. PLA/CB conductive nanocomposite thermoplastic materials could be ideal feedstock materials for AM FFF 3D printing processes, and therefore systematic investigations are required to highlight the fundamental process-structure-property relationship.

CPC multifunctional filaments with electrical properties have been fabricated and utilized for FFF 3D printing towards biomedical sensors [32], piezoresistive strain sensors [33], actuators [34] and resistors for Joule-heating [35]. Specifically, Joule resistive heating or Ohmic heating is the effect when an applied electric current pass through an electrical conductor, resulting in heat generation. In principle, when voltage difference is applied between two points of a conductive network, an electric field is being generated, which accelerates charge carriers in the field direction, resulting in higher kinetic energy. Then, electrons collide within the conductor's ions and the particles are being scattered. Under this atomic scale electronic charge carrier transport mechanism, electrical energy is converted to thermal energy [36]. From Joule's first law, it arises that the consumed power of heating, which is generated by an electrical conductor, is proportional to the electrical resistance and the square of the current: $\mathrm{P}=\mathrm{I}^{2} \cdot \mathrm{R}$, where P: Power (W), I: Current (A) and R: Resistance (Ohm). According to the Ohmic conductor model, the transduction of electrical power into Joule-heating is given by the relation $\mathrm{P}=\mathrm{V}^{2} / \mathrm{R}$, where $\mathrm{V}(\mathrm{V})$ denotes the applied input voltage, or otherwise known as voltage bias (Vbias). Nowadays, there are several applications of resistive heating with numerous solutions already existing in the market [37], while 3D printed CPCs could be ideal lightweight candidates for advanced applications.

Aumnate et al. reported on reinforcing Polypropylene (PP) with graphene-polylactic acid microcapsules for FFF 3D printed objects. At low graphene loading (0.75 wt.\%), the $3 \mathrm{D}$ printed construct showed neither shrinkage nor warping. Graphene nanocomposite 3D printed constructs with 70\% infill exhibited higher mechanical performance, when compared to neat PP [38]. In another study related to isotactic polypropylene/graphene 3D printed nanocomposites, the authors reported that the presence of graphene induced shear thinning during extrusion (improved processability aspect), while at $5 \%$ and $10 \%$ of graphene loading, the storage modulus decreased considerably; namely nearly $50 \%$ for the $10 \%$ loading [39]. Recently, Lei et al. reported on FFF 3D printed carbon black (CB)/polypropylene composites with excellent microwave absorption performance [40], while Kwok et al. reported on PP/CB electrically conductive nanocomposite filaments for FFF 3D-printed circuits and sensors [41], both focusing only onto the electrical properties of the 3D printed composites. CPC filaments for FFF 3D printing and electrically conductive 3D objects have been reported so far, especially focusing on the electrical and thermoelectric properties of 3D printed nanocomposites, as reported elsewhere [42]. In the 3D printing technology, Polyethylene/graphene nanocomposites have been investigated for heat diffusion applications and advanced electronic devices [43], Low Density Polyethylene (LDPE) composite filled with copper particles and Sn95Ag4Cu1 has been studied for compressive sensing applications [44], while Acrylonitrile Butadiene Styrene/graphite has been examined for electrochemical sensor development [45]. PLA/CB studied in this work has been employed in electrochemical sensors [46], electronic components and circuits [47] and electrodes [48], with none of these studies focusing on the characterization of the material, which was commercial in some cases [47]. Additionally, the material developed did not 
have multifunctional performance, with research focusing to cope with the requirements of specific applications in 3D printing.

Although there is a lot of interest in literature for the development of conductive materials in AM in general, the effect of specific fillers on properties (mechanical, thermal, electrothermal, antibacterial, etc.) of various matrices is not yet reported. Additionally, the methodology for the preparation of conductive nanocomposites suitable for 3D printing has many technological aspects that need to be investigated, in order to be optimized. Finally, in literature the electrical conductivity is studied in different composites, but the development of nanocomposites suitable for $3 \mathrm{D}$ printing with multifunctional performance is still not available in literature. To the best of the author's knowledge, a detailed study of PLA/CB conductive nanocomposites focusing on the electrical percolation threshold as a function of the filler loading, as well as a detailed static mechanical and thermomechanical analyses of the 3D printed samples has not been reported yet.

In this work PLA/CB nanocomposites at 0.5, 1.0, 2.5 and $5.0 \mathrm{wt} . \%$ filler loadings were produced by a thermomechanical melt mixing process, to produce $1.75 \mathrm{~mm}$, suitable for $3 \mathrm{D}$ printing. Neat PLA and PLA/CB nanocomposites in filament form were then utilized as feedstock material for the specimens FFF 3D printing manufacturing step. FFF process is based on the extrusion principle. All 3D printed specimens were then fully investigated for their static and thermomechanical properties, according to international standards. The highest reinforcement regarding the tensile properties was found for the PLA/CB of $0.5 \mathrm{wt} . \%$, while in the flexural, impact and microhardness tests, the highest values were observed for the PLA/CB $2.5 \mathrm{wt} . \%$ nanocomposites.

The PLA/CB nanocomposites were then fully characterized in terms of their electrical conductivity. The PLA/CB $5.0 \mathrm{wt} . \%$ also performed as a Joule heater device, as well as a conductive circuitry resistor, evaluating the multi-functional nature of the $3 \mathrm{D}$ printed nanocomposites prepared and investigated in this work. The PLA/CB nanocomposites exhibited an electrically conductive behavior from the $2.5 \mathrm{wt} . \%$ loading. The electrical conductivity increased with the increase of the filler loading up to the highest loading of $5.0 \mathrm{wt} . \%$ prepared in this work. The highest electrical conductivity $(\sigma)$ was obtained at the "cross-layer" direction for the PLA/CB $5.0 \mathrm{wt} . \%$ nanocomposite $(\sim 0.9 \mathrm{~S} / \mathrm{m})$, which also exhibited an electrothermal "Joule-heating" function upon being exposed to a voltage bias (Vbias) of $15 \mathrm{~V}$. Scanning Electron Microscopy (SEM) fractographic analyses revealed the underlying fracture mechanisms of the tensile tested specimens, while Raman spectroscopy revealed the graphitic nature of CB and the PLA matrix characteristic spectroscopic fingerprints. Thermogravimetric Analysis (TGA) showed the effect of filler loading on the thermal properties of the fabricated nanocomposites. Enhanced thermal stability was observed for all the PLA/CB nanocomposites prepared in this work. Finally, the antimicrobial properties of the 3D printed PLA/CB nanocomposites of this work were examined with a screening process against $E$. coli and S. aureus bacteria. A mild antibacterial performance was found only at the nanocomposite with the highest filler loading. Overall, the FFF 3D printed PLA/CB CPCs manufactured herein verified their multifunctional nature and could be employed in various applications as flexible conductors, resistors for circuitry, as well as piezoresistive sensors for strain sensing, etc., while their antibacterial performance is an additional asset in biomedical, hygienic and other health-related applications.

\section{Materials and Methods}

\subsection{Materials}

Polylactic Acid (PLA), the matrix material in this study, was of 3052D grade, with molecular weight of $116,000 \mathrm{~g} / \mathrm{mol}$. It was procured from Plastika Kritis SA (Heraklion, Greece) in the form of coarse powder. A highly conductive Carbon Black (CB) grade (CNERGY Super P Conductive Carbon Black) was received from Nanografi Nanotechnology AS (Tallinn, Estonia) as a mechanical reinforcing and electrically conductive agent. 


\subsection{Fabrication of Filaments and FFF 3D Printing Process of PLA and PLA/CB Nanocomposites}

PLA and CB filler mixtures were prepared under five (5) different filler ratios. Mixing percentages were of $0.1 \%, 0.5 \%, 1.0 \%, 2.5 \%$ and $5.0 \%$ in a weight to weight $(w / w)$ percentage ratio (wt.\%). Pure PLA was also produced as a reference material for all nanocomposite materials. Adequate quantity of each material was weighted and mixed using mechanical mixing equipment. To ensure the lack of humidity in all material mixtures/formulations, drying procedures were implemented throughout the process steps. Namely, PLA coarse powder was dried for $24 \mathrm{~h}$ at $50{ }^{\circ} \mathrm{C}$ using a laboratory oven. Further drying was applied after the mixing process for all materials, in this case for $5 \mathrm{~h}$ at $50^{\circ} \mathrm{C}$.

Extruded filaments with $1.75 \mathrm{~mm}$ in diameter suitable for FFF 3D printing were produced, with a melt mixing thermomechanical process, using a 3D Evo (3D Evo B.V., Utrecht, the Netherlands) single screw extruder. Specifically, 3D Evo Composer 450 was employed, which features an optimized and appropriate screw for mixing/compounding purposes during the consecutive extrusion procedure. Its chamber has four (4) heating zones, and the device also features a filament diameter real-time measurement system. In this way, produced filament quality control is implemented and monitored in realtime with the extrusion/production process, determining the filament diameter accuracy and adjusting in real-time the extrusion parameters automatically, to maintain the required accuracy tolerances. The extruded filament diameter was measured to be in all cases $1.68 \mathrm{~mm} \pm 0.07 \mathrm{~mm}$, which is an adequate accuracy for consistent and high-quality $3 \mathrm{D}$ printing. All the produced materials, i.e., pure PLA and the five different PLA/CB nanocomposites were processed under the same extrusion parameters. Temperatures were set to each heating zone; $175^{\circ} \mathrm{C}$ at heat zone 4 (closer to hopper), $205^{\circ} \mathrm{C}$ at heat zone 3 and 2 (middle stage) and $195^{\circ} \mathrm{C}$ at heat zone 1 (closer to extruder's nozzle). Screw rotational speed was set to $7.4 \mathrm{rpm}$ (extruder's range is $2.5-15 \mathrm{rpm}$ ) and the built-in winder rotational speeds were automatically adjusted to achieve the requested diameter. Additional quality control tests were also manually conducted with random diameter measurements to the filament diameter, using a high-quality electronic caliper and optical quality control for defects. The produced filaments were then dried at the same conditions, before being used to produce the various 3D printed specimens. The filament diameter was inserted in the 3D printer slicer software, to calculate the necessary feed rate during the entire 3D printing process.

Fused Filament Fabrication (FFF) was selected to manufacture the neat PLA as well as the PLA/CB nanocomposite specimens. An Intamsys (Shanghai, China) 3D Printer was chosen, using an extruder's nozzle of $0.4 \mathrm{~mm}$. Specifically, the Intamsys HT 3D printer was used for specimens' fabrication through AM. Initial trails were performed to obtain the optimum FFF 3D printing parameters (set of parameters) for high quality $3 \mathrm{D}$ printing for the pure PLA and the PLA nanocomposites. For the mechanical characterization, the electrical conductivity and the antibacterial test measurements, all 3D geometric models were designed employing the 3D design Autodesk ${ }^{\circledR}$ Fusion $360^{\text {TM }}$ (Autodesk ${ }^{\circledR}$, Inc., San Rafael, CA, USA) software platform. 3D geometry was finally exported to Standard Tessellation Language (STL) files from the Computer Aided Design (CAD) software platform.

Figure 1 shows the flow chart illustrating the methodology followed in this study to produce and characterize the PLA and the PLA/CB nanocomposites. Figure 2 summarizes the complete list of the $3 \mathrm{D}$ printing parameters used to fabricate the specimens in the 3D printer, after an initial screening optimization approach. All other parameters, not mentioned in Figure 2 were set to their default values, in the Intamsuite software tool (after choosing PLA as the reference material in the 3D printer software tool), which was used as the slicer software tool for this study. 


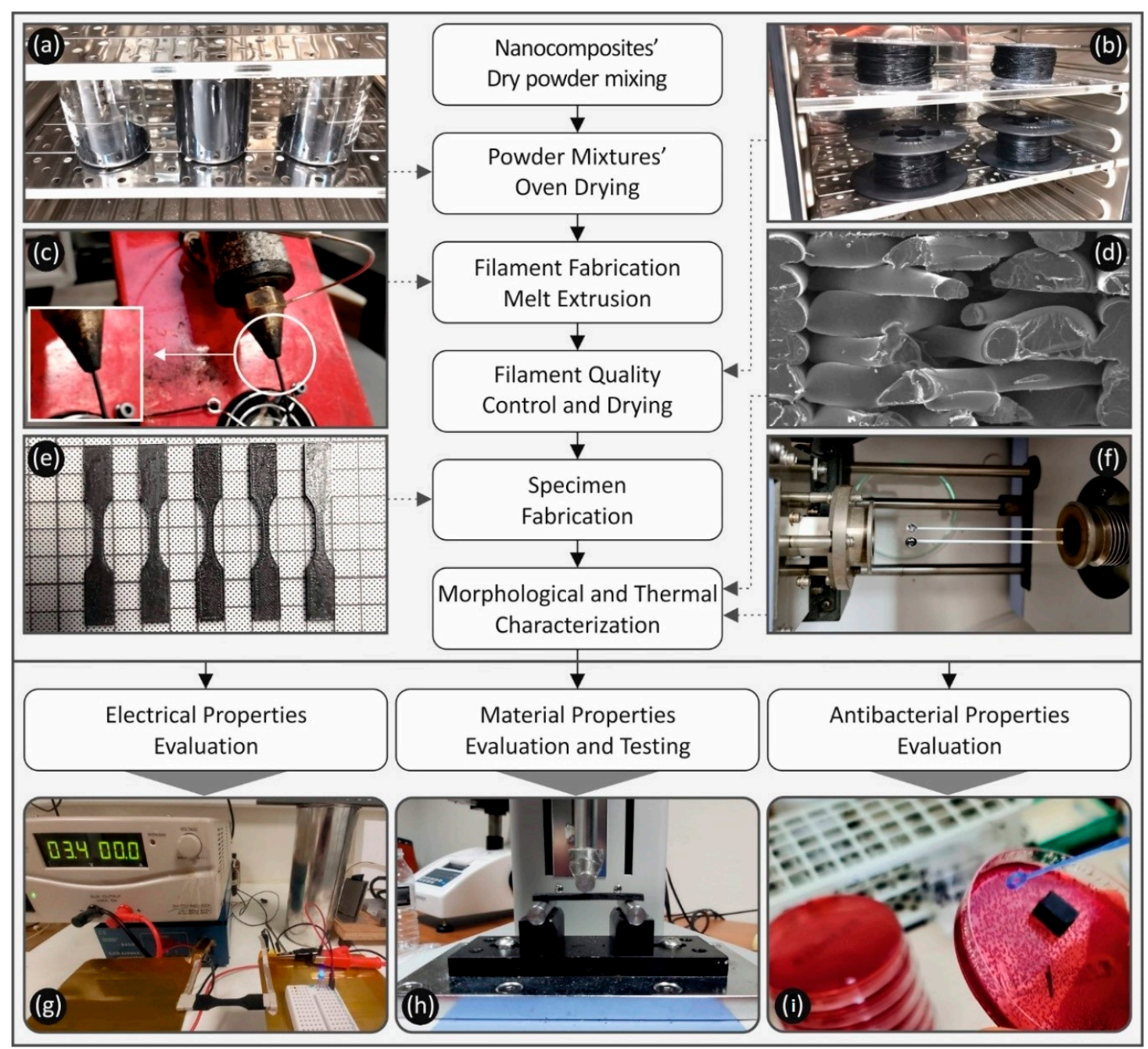

Figure 1. Flow chart illustrating the different steps to manufacture the filaments, as well as the 3D printed specimens and the characterization of the nanocomposites fabricated in this work: (a) powders' drying process, (b) filaments' drying process, (c) filaments' extrusion process, (d) tensile test specimens' morphological characterization on SEM, (e) tensile test specimens, (f) thermogravimetric analysis on the nanocomposites, (g) electrical conductivity measurements on the specimens, (h) flexural test on specimen, (i) antibacterial screening process on the nanocomposites.

\begin{tabular}{llll|}
\hline Parameters & Value & Units \\
\hline Printing orientation & 45 & Deg. \\
Layer thickness & 0.200 & $\mathrm{~mm}$ \\
Bed temperature & 85 & ${ }^{\circ} \mathrm{C}$ \\
Nozzle temperature & 255 & ${ }^{\circ} \mathrm{C}$ \\
Number of perimeters & 2 & \\
Top solid layers & 4 & $\mathrm{~mm} / \mathrm{s}$ \\
Bottom solid layers & 4 & \\
Fill Density & 100 & 40 & \\
Travel speed & &
\end{tabular}

Figure 2. The optimum FFF 3D printing parameters followed in this study and set up to slicer software to manufacture the neat PLA, as well as the PLA/CB different nanocomposite specimens. 


\subsection{Characterization Techniques}

Raman spectroscopy was performed for the pure PLA and CB, as well as the PLA/CB nanocomposite 3D printed specimens, using a Labram HR-Horiba (Kyoto, Japan) scientific micro-Raman system. An optical microscope equipped with a $50 \times$ long working distance objective was utilized for delivering the excitation light and collecting the back-scattering Raman activity. An $\mathrm{Ar}^{+}$ion laser line $(514.5 \mathrm{~nm})$ at $1.5 \mathrm{~mW}$ power at the focal plane was utilized for the Raman excitation. All acquired spectra shown in this work have been treated with a baseline correction through subtraction of a linear or polynomial fit of the baseline from the raw spectra, to remove tilted baseline variation caused by various noises, i.e., fluorescent background, etc.

Thermogravimetric Analysis (TGA) was carried out for the 3D printed samples, namely the neat PLA, as well as the PLA/CB nanocomposites in oxygen atmosphere. The measurements were performed with a Perkin Elmer Diamond TG/TDA (Waltham, MA, USA) with a heating cycle from $30^{\circ} \mathrm{C}$ to $550{ }^{\circ} \mathrm{C}$ with a heating step of $10^{\circ} \mathrm{C} / \mathrm{min}$.

SEM characterization was used to analyze the nano/micro structuring of both the 3D printed specimens' side surface, as well as the fractured surfaces of 3D printed tensile specimens. SEM microstructural analyses were performed using a JEOL JSM 6362LV (Jeol Ltd., Norwood, MA, USA) electron microscope in high-vacuum mode at $20 \mathrm{kV}$ acceleration voltage. Prior to SEM investigations, samples were sputter-coated with gold (Au) to avoid charging effects.

The through-thickness (through-layer) as well as in-plane (cross-layer) electrical conductivity of 3D printed PLA/CB nanocomposite samples was determined using squareshaped samples of 10.0 by $10.0 \mathrm{~mm}^{2}$ and $3.2 \mathrm{~mm}$ thickness. Ag paste (room temperature fast curing curing/drying silver paste received from Agar scientific, Stansted, Essex, United Kingdom) was applied in the appropriate surfaces to contact the samples for the respective through-layer and cross-layer conductivity measurements. Afterwards, 2-point probe electrical resistance measurements were performed with an Agilent Multimeter (Agilent $34401 \mathrm{~A} 6 \frac{1}{2}$, Agilent, Santa Clara, CA, USA) to derive the electrical resistivity $(\rho)$ and conductivity $(\sigma)$, respectively, considering the specific sample dimensions.

Thermal images demonstrating the nanocomposite samples capability to function as electrothermal Joule heating materials were captured with a Seek Thermal Compact PRO Compact XR Imaging Camera IR Infrared Imager (Santa Barbara, CA, USA) with a temperature working window of -40 to $330{ }^{\circ} \mathrm{C}$.

Specimens were tested for the determination of their mechanical properties in room temperature $\left(23^{\circ} \mathrm{C}\right)$. Specifically, quasi-static tensile test experiments were carried out at room temperature $\left(23^{\circ} \mathrm{C}\right)$ following the ASTM D638-02a international standard. According to the standard, a type $\mathrm{V}$ specimen of $3.2 \mathrm{~mm}$ thickness was chosen, and a total of six (6) specimens were manufactured and tested for each case. An Imada MX2 (Imada Inc., Northbrook, IL, USA) tension/flexure test apparatus in tensile mode using standardized grips was utilized to carry out the tensile test experiments at an elongation rate of $10 \mathrm{~mm} / \mathrm{min}$.

Flexural (three-point bending) tests were carried out also at room temperature conditions on 3D printed specimens ( $64.0 \mathrm{~mm}$ length, $12.4 \mathrm{~mm}$ width and $3.2 \mathrm{~mm}$ thickness), according to the ASTM D790-10 international standard (three-point bending test with $52.0 \mathrm{~mm}$ support span). Flexural test specimens were manufactured with the same 3D printing parameters as for the tensile test specimens. An Imada MX2 machine in flexural mode setup was employed for the three-point bending tests. The same speed of $10 \mathrm{~mm} / \mathrm{min}$ was set for the testing procedure, and a total of six (6) specimens were manufactured and tested for the neat PLA, as well as PLA/CB nanocomposites.

Impact tests were performed according to the ASTM D6110-04 international standard. Specimens were 3D printed with the following dimensions: $80.0 \mathrm{~mm}$ (length) by $8.0 \mathrm{~mm}$ (width) by $10.0 \mathrm{~mm}$ (thickness). Six (6) notched specimens in total were tested using a Terco MT 220 (Terco, Kungens Kurva, Sweden) Charpy's impact apparatus. Release height of the apparatus hammer was the same for all the experiments $(367 \mathrm{~mm})$. 
Microhardness measurements were conducted according to the ASTM E384-17 international standard. The specimens' surface was fully polished before each set of measurements. An Innova Test 300-Vickers (Innovatest Europe BV, Maastricht, The Netherlands) apparatus was employed, while the applied force was set to $100 \mathrm{gF}$ and duration of $10 \mathrm{~s}$ was selected for indentation. Imprints were measured under six (6) different specimens for each one of the PLA and PLA/CB nanocomposites.

Dynamic Mechanical Analysis (DMA) was performed using a TA Instruments DMA850 instrument (New Castle, DE, USA). Samples were 3D printed in dimensions of length 58.0-60.0 $\mathrm{mm}$, width 14.0-15.0 $\mathrm{mm}$ and thickness $2.7-3.2 \mathrm{~mm}$. Due to the samples having rough side edges from the FFF manufacturing process, all samples were polished in two steps using 240 and 400 grain sandpaper under water flow. Prior to testing, samples were dried at a temperature of $35^{\circ} \mathrm{C}$ for a minimum of $48 \mathrm{~h}$. The DMA testing procedure consisted of a temperature ramp from room temperature to $130^{\circ} \mathrm{C}$ (and in some cases up to $135^{\circ} \mathrm{C}$ ), at a rate of $3^{\circ} \mathrm{C} \mathrm{min}^{-1}$. Testing was conducted using the three-point bending fixture. Samples were preloaded to $0.1 \mathrm{~N}$. A sinusoidal displacement was applied to the samples with a constant amplitude of $30.0 \mu \mathrm{m}$ and a frequency of $1.0 \mathrm{~Hz}$ throughout the tests. Data was collected by the instrument at a sampling rate of $0.33 \mathrm{~Hz}$. The recorded parameters were the storage modulus, loss modulus, $\tan \delta$, temperature, time and oscillation angular frequency.

The antibacterial performance of the nanocomposites of this work, was investigated with the agar well diffusion method [49] implemented in a microbiological lab, for two different bacteria, i.e., E. coli and S. aureus. Petri dishes (850 $\mathrm{mm}$ in diameter) with suitable bacterium growth material (MC.2, C.010066 for the E. coli and Chapman, C.010068 for the $S$. aureus), were employed for the tests. Two rectangular specimens (one for each bacterium) of $12.4 \mathrm{~mm}$ by $12.4 \mathrm{~mm}$ and $3.2 \mathrm{~mm}$ height were 3D printed with the same parameters described above, from pure PLA and each one of the different nanocomposite materials prepared in this work. The bacteria were inoculated in the corresponding Petri dishes and the specimens were placed near the center of the Petri dishes, with one specimen placed in each Petri dish. The Petri dishes were placed in an oven at $37^{\circ} \mathrm{C}$ for $24 \mathrm{~h}$ for the diffusion of the antimicrobial agent into the nanocomposite and inhibit germination and growth of the test microorganism. Then, the width of the inhibition growth zone was measured.

\section{Results and Discussion}

\subsection{Raman Analysis of PLA, CB and PLA/CB Nanocomposites}

Figure 3 shows the Raman spectrum of PLA, CB and PLA/CB nanocomposite (at $2.5 \mathrm{wt}$ \% of filler loading) at the spectral region $300-3300 \mathrm{~cm}^{-1}$. It is worth mentioning that the Raman spectra of PLA/CB nanocomposites at higher filler loadings were governed by the carbon nanoadditive "characteristic" spectroscopic responses, which are highly resonant to the $532 \mathrm{~nm}$ laser excitation source, without any visible peaks arising due to the PLA polymeric matrix. It can be very clearly observed in the CB and in the PLA/CB spectra the characteristic D-band and G-band peaks of CB graphitic carbon allotrope materials, centered at ca. $1345 \mathrm{~cm}^{-1}$ and $1565 \mathrm{~cm}^{-1}$, respectively [50]. 


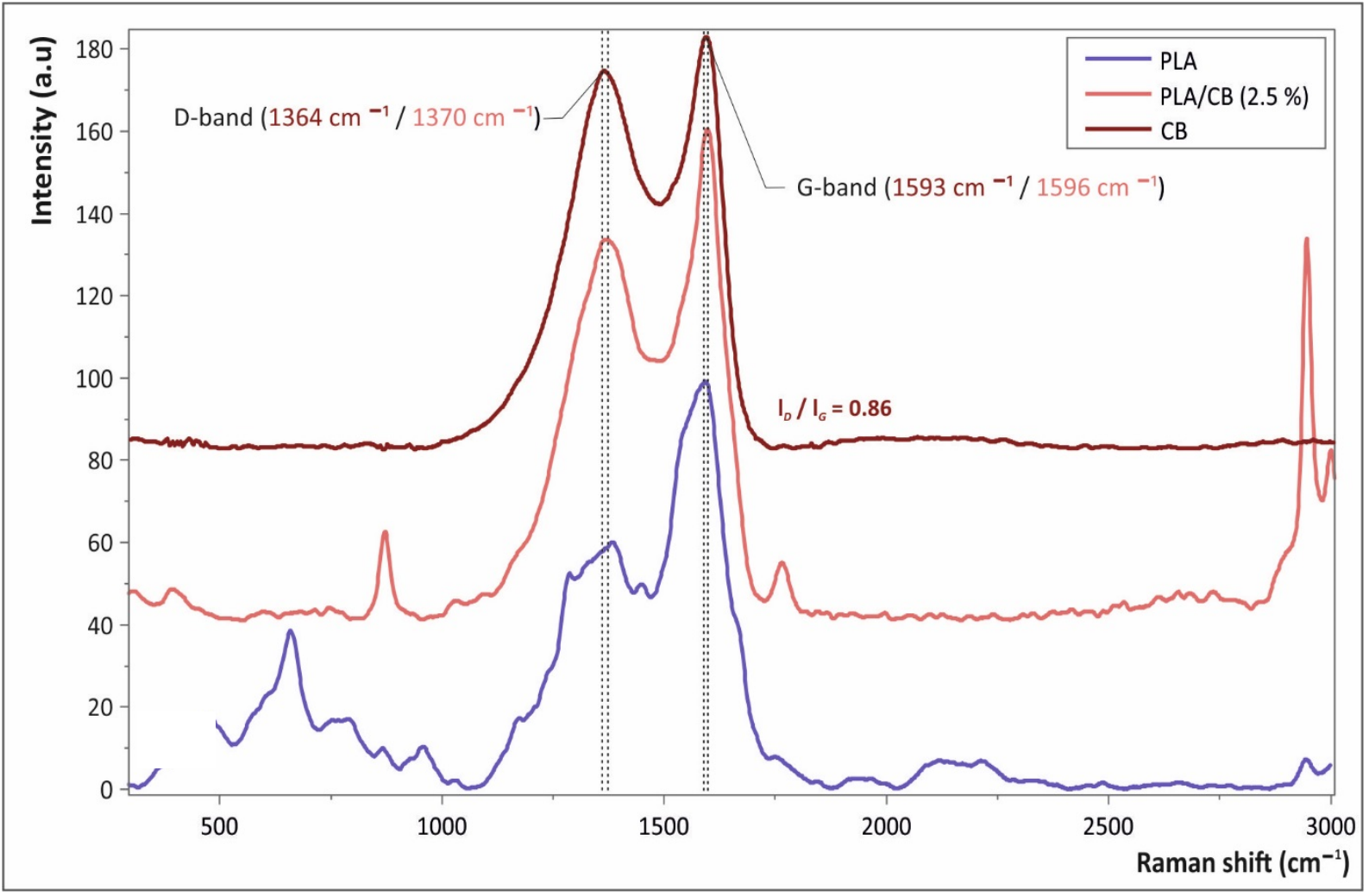

Figure 3. Raman spectra of PLA, CB and PLA/CB nanocomposite (at 2.5 wt.\% of filler loading) at the spectral region $300-3300 \mathrm{~cm}^{-1}$.

The graphitization and degree of crystallinity of carbon nanoallotrope materials have been previously correlated to the relative intensity ratio of the corresponding D and Gbands $\left(I_{\mathrm{D}} / I_{\mathrm{G}}\right)$ [51]. Specifically, the $I_{D} / I_{G}$ intensity band ratio of CB utilized as electrically conductive and mechanical reinforcing filler in this study is 0.86 (indicated also as inset in the corresponding Raman spectrum in Figure 3). For the PLA/CB spectrum, there are characteristic peaks attributed to the PLA matrix, as well as the graphitic fingerprints of CB (indicated with dashed lines). Specifically, the PLA characteristic fingerprints attributed to the PLA macromolecular chains' chemistry (backbone chain, side and end-terminal groups) are the following: 465 and $589 \mathrm{~cm}^{-1}$ (C-O-C vibration), $655 \mathrm{~cm}^{-1}$ (C=O stretching vibration), $936 \mathrm{~cm}^{-1}$ (C-COO vibration), 1249 and $1320 \mathrm{~cm}^{-1}$ ( $\mathrm{CH}$ deformation vibration), $1361-1385 \mathrm{~cm}^{-1}\left(\mathrm{CH}_{3}\right.$ deformation vibrations of PLA), $1587-1596 \mathrm{~cm}^{-1}$ (asymmetric $\mathrm{C}=\mathrm{O}$ stretching vibrations of carboxylate groups of PLA) and $2947 \mathrm{~cm}^{-1}\left(\mathrm{CH}_{3}\right.$ symmetric and asymmetric stretching vibration) [21], while only few of them are visible in the PLA/CB nanocomposite, due to the intense peaks of $\mathrm{CB}$.

\subsection{Thermogravimetric Analysis (TGA) of 3D Printed Neat PLA and PLA/CB Nanocomposites}

TGA investigations revealed the critical degradation temperature of the neat PLA, as well as the PLA/CB nanocomposites under oxygen, simulating thus any plausible degradation may occur during the melt mixing filament extrusion process and the $3 \mathrm{D}$ printing consecutive process for the specimen's manufacturing/preparation.

Figure 4 shows the TGA (Figure $4 a$ ), and the DTG (Figure $4 \mathrm{~b}$ ) corresponding graphs of the $3 \mathrm{D}$ printed neat PLA, as well as the PLA/CB nanocomposites in the temperature window of $50-550{ }^{\circ} \mathrm{C}$. Three distinct thermal windows can be observed. The first one is up to $\sim 270{ }^{\circ} \mathrm{C}$ that all materials exhibited thermal stability without any observed weight loss (\%). From $270{ }^{\circ} \mathrm{C}$ up to $374{ }^{\circ} \mathrm{C}$, there is a second temperature window, corresponding to the materials' thermal degradation and decomposition with the onset temperature 
of decomposition $\left(\mathrm{T}_{\mathrm{on}}\right)$ at $273{ }^{\circ} \mathrm{C}$. In the third temperature window $\left(>370-380{ }^{\circ} \mathrm{C}\right.$ up to $\left.550^{\circ} \mathrm{C}\right)$, all the PLA substance for all samples' formulation has been fully decomposed.
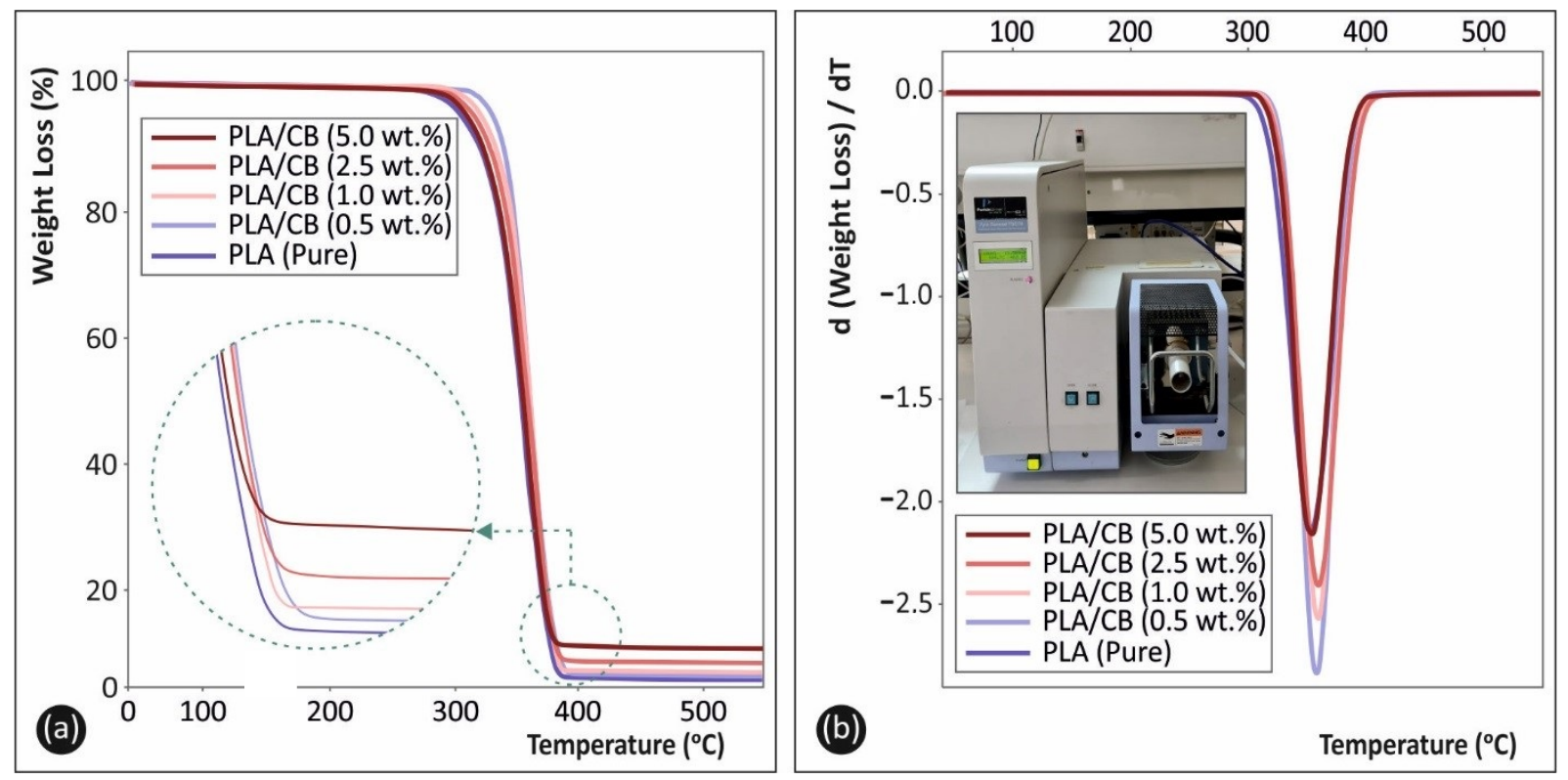

Figure 4. (a) Thermogravimetric Analysis (TGA) and (b) Differential Thermogravimetry (DTG) plots of 3D printed neat PLA and PLA/CB nanocomposites (inset TGA scan in Figure 4a shows the nanocomposites' remnant due to CB, as well as the increased thermal stability induced by the CB nanofiller at all particle loadings).

The inset TGA scan in Figure 4a shows more precisely (i) the remnant material attributed to the $\mathrm{CB}$ nanofiller above $370-380{ }^{\circ} \mathrm{C}$, as well as (ii) the slightly increased materials' (PLA) thermal stability induced by the CB nanofiller at all particle loadings, due to the $C B$ thermal properties. It is worth mentioning that all materials exhibited similar decomposition characteristics, especially for the behavior of the polymer substance used as the matrix, corroborating the extremely high control of the filament extrusion and $3 \mathrm{D}$ printing process upon applying the same parameters and resulting, thus, in the same thermal treatment history for all the 3D printed/manufactured samples. The overall thermal stability of the 3D printed specimens does not seem to be impacted; however, it is even enhanced by the addition of the CB, which nanoparticles are well-known for their thermal conductivity; endowing an enhanced thermal degradation performance of the PLA/CB nanocomposites.

\subsection{Scanning Electron Microscopy of 3D Printed Sample's Side Surface}

SEM microstructural analyses of the 3D printed tensile test samples' side surface morphology (at two different magnifications) were performed to examine the 3D printed specimens' external structure, revealing more precisely the 3D printed samples' interlayer fusion. Figure 5 depicts the side surface morphology of neat PLA and PLA/CB 3D printed nanocomposites at two different magnifications: namely neat PLA (Figure $5 a, b)$, as well as 0.5 wt.\% (Figure 5c,d), 2.5 wt.\% (Figure 5e,f) and 5.0 wt.\% (Figure $5 \mathrm{~g}, \mathrm{~h}$ ) nanocomposites. For all samples, an excellent interlayer fusion can be observed between the different AM layers, apart from the PLA/CB at $5.0 \mathrm{wt} . \%$ (Figure $5 \mathrm{~g}, \mathrm{~h}$ ) where some defects and discontinuities can be seen. For 3D printed AM specimens, the fusion between the layers affects the stress transfer between the 3D printed filaments' layers, resulting in an optimum interfacial shear strength of the adjacent layers and filaments of the 3D printed parts. This is a prerequisite towards high mechanical performance 3D printed nanocomposite parts, exhibiting an enhancement and reinforcement mechanism due to the presence of the nanofillers. 

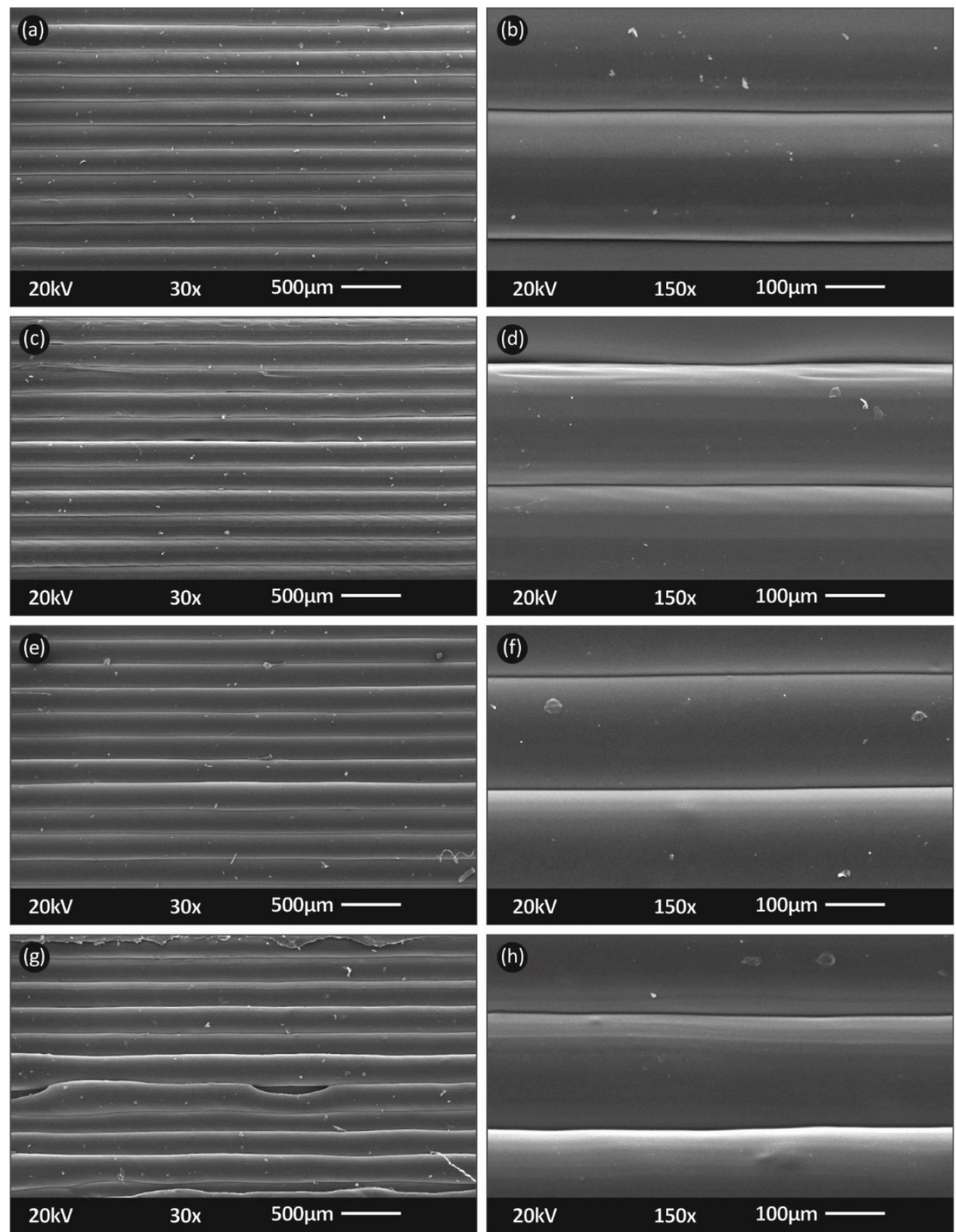

Figure 5. Side surface morphology of 3D printed tensile test specimens of neat PLA (a,b), as well as PLA/CB with 0.5 wt.\% (c,d), 2.5 wt.\% (e,f) and 5.0 wt.\% (g,h) CB filler loading.

The high quality of the interlayer fusion for the majority of the samples in this study indirectly proves: (i) the optimum 3D printing parameters initially determined and applied in this study, as well as (ii) homogeneously dispersed CB nanofiller in the PLA extruded filaments, since nanoparticle aggregation in the feedstock filament could significantly affect the 3D printing quality introducing inhomogeneities, defects, discontinuities, etc. It is well-known that the existence of micro-aggregates in the filament feedstock could impart structural defects in the final 3D printed samples, since it can affect the polymer melt rheological properties, causing thus nozzle clogging phenomena. In the 3D printed nanocomposites herein, it is worth mentioning that only in the case of PLA/CB (5.0 wt.\%) nanocomposite the SEM side surface microstructure reveals some inhomogeneity in the 3D printed layer thickness, as well as some discontinuities and defects/holes between the layers. This is most likely attributed to the increased polymer melt viscosity hampering during the 3D printing filamentous extrusion process. 


\subsection{Electrical Conductivity of 3D Printed PLA/CB Nanocomposites}

Figure 6a illustrates schematically the two-probe electrical resistance measurements performed in this study to determine the electrical conductivity of the 3D printed PLA/CB nanocomposites. The electrical conductivity has been derived in two different directions of the 3D printing process, to examine any plausible anisotropic electrical conductivity behavior, typically known in additive manufacturing produced specimens.

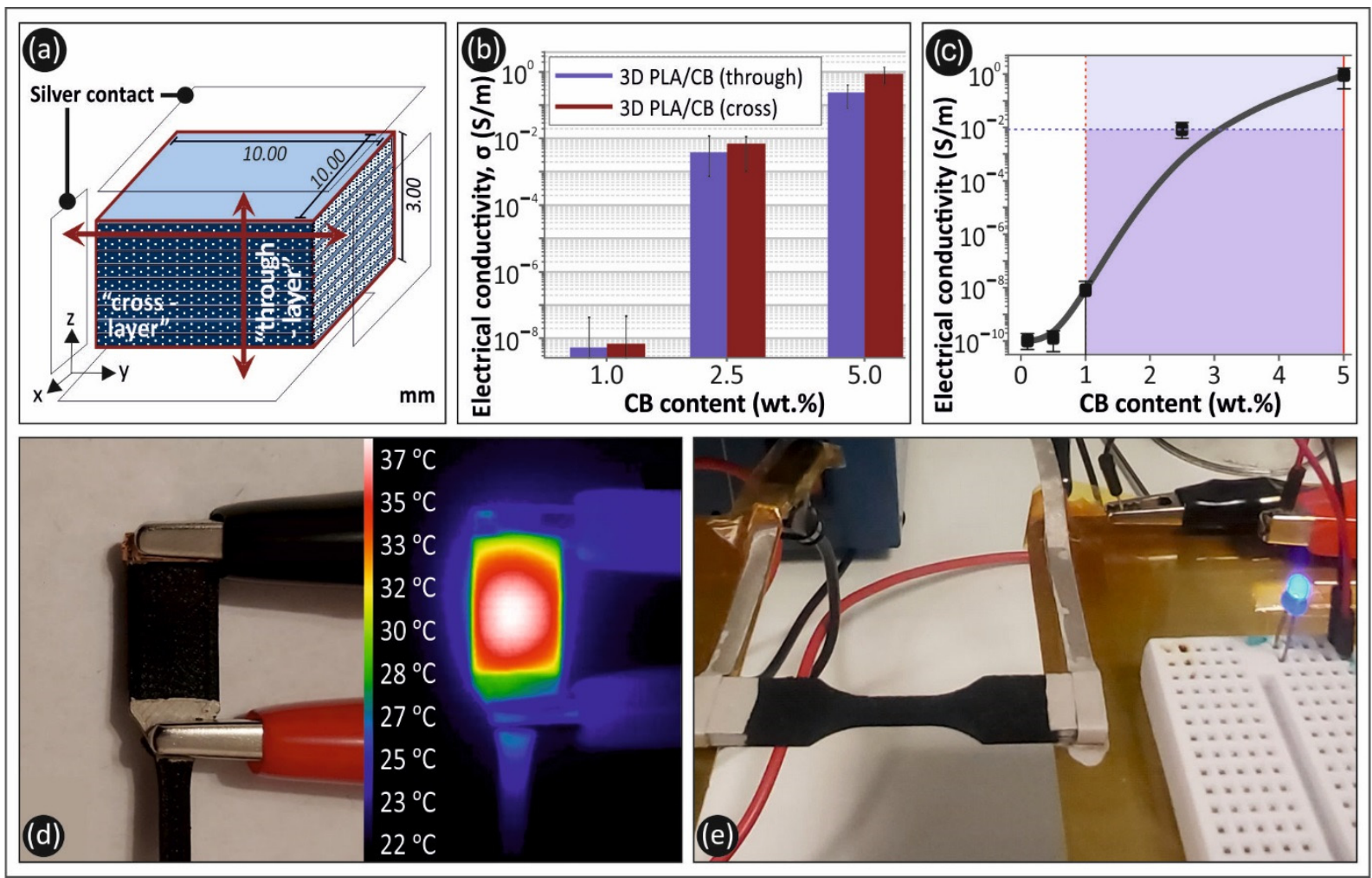

Figure 6. (a) schematic illustration of the electrical conductivity measurements for the 3D printed PLA/CB CPC specimens at two different directions; (b) electrical conductivity of the 3D printed conductive nanocomposites at 1.0, 2.5 and 5.0 wt.\% CB content (at two different measurement directions; namely the "through layer" and the "cross-layer"), as well as (c) a detailed plot of the PLA/CB electrical conductivity at various filler loadings (0.1, 0.5, 1.0, 2.5 and 5.0 wt.\% CB filler content), demonstrating the PLA/CB CPC percolation curve; (d) a real sample digital image (with the two deposited electrodes), as well as an IR-T image showing the electrothermal Joule heating performance of the PLA/CB (5.0 wt.\%) nanocomposite at an applied DC Vbias of $15 \mathrm{~V}$; (e) LED in operation when current is passing through a PLA/CB (5.0 wt.\%) nanocomposite in a closed circuit applying a Vbias of $15 \mathrm{~V}$.

Figure $6 \mathrm{~b}$ shows the electrical conductivity of the PLA/CB nanocomposites at 1.0, 2.5 and $5.0 \mathrm{wt} . \% \mathrm{CB}$ content, all of which are above the electrical percolation threshold, exhibiting typical "conductor" characteristics (the $1.0 \mathrm{wt} . \%$ is slightly above the percolation threshold; the samples are not showing a fully insulating behavior, which is expected when measuring a polymeric matrix). Conductivity values of the percolated samples are given for the two different measurement directions: namely the "through layer" or through thickness, as well as the "cross-layer" or in-plane direction. It can be easily observed that for all 3D printed PLA/CB CPCs, the cross-layer conductivity values are a bit higher than the through thickness ones. This is a direct proof that the 3D printed nanocomposite samples exhibit "anisotropic" electrical transport properties induced by the FFF process. This finding is in good agreement with other 3D printed CPCs reported elsewhere [32]. Namely, the highest conductivities were determined for the $5.0 \mathrm{wt} . \%$ PLA CPC nanocomposites, i.e., $0.3 \pm 0.2 \mathrm{~S} / \mathrm{m}$ and $0.9 \pm 0.5 \mathrm{~S} / \mathrm{m}$ for the "through layer" and "cross-layer" directions, respectively. Nevertheless, the slight difference in the electrical conductivity values at the two directions reveal a high quality and extent of interlayer fusion between the 3D printed 
consecutive layers: resulting in an almost homogeneous and identical "bulk" electrical conductivity behavior.

Figure $6 \mathrm{c}$ represents the electrical conductivity percolation curve for the 3D printed PLA/CB nanocomposites at different filler loadings; namely 0.1, 0.5, 1.0, 2.5 and $5.0 \mathrm{wt} . \%$ $\mathrm{CB}$ filler content (all values correspond to the "cross-layer" conductivity). As it can be observed, a conductive behavior for the PLA/CB nanocomposites is achieved at CB filler loading of $1.0 \mathrm{wt} . \%$, increasing further with the increase of the filler content. The percolation curve exhibits a typical "S-shaped", otherwise known as sigmoidal curve shape, which is typical for randomly dispersed conductive fillers in an insulating polymer matrix [52].

Figure $6 \mathrm{~d}$ depicts the Joule heating electrothermal performance of the highest electrically conductive PLA/CB nanocomposite sample (5.0 wt.\%), upon applying a DC voltage bias ( $\mathrm{V}_{\text {bias }}$ ) of $15 \mathrm{~V}$ (using a conventional DC power supply). The infrared thermography (IR-T) image has been captured after a time duration of $t=30 \mathrm{~s}$ that the DC voltage has been applied at the two terminals of the specimen. As it is shown in the figure, the sample's surface temperature raises at approximately $\sim 35^{\circ} \mathrm{C}$.

The exploitation of the electrothermal, otherwise known as "Joule-heating" effect, has been widely studied by the research community for different scopes and in various sectors utilizing conductive nanoparticle nanocomposites [53]. Figure 6e represents the operation of an LED device when current is passing through a $5.0 \mathrm{wt} . \%$ filled PLA/CB nanocomposite in a closed circuit, upon applying a voltage bias $\left(V_{\text {bias }}\right)$ of $15 \mathrm{~V}$, demonstrating the potential use of the PLA/CB nanocomposites as lightweight resistors. Figure $6 \mathrm{~d}$,e represent two significant demonstration scenarios for various practical applications of the 3D printed PLA/CB nanocomposites, arising from the electrically conductive nature of the 3D printed PLA/CB samples induced by the dispersed CB conductive phase. These two representative experiments reveal the multifunctional character of the 3D printed PLA conductive nanocomposites fabricated in this study, which could enable practical future applications such as flexible circuitry, flexible wires and flexible and wearable sensors, expanding further the well-known and emerging field of 3D printed electronics.

\subsection{Mechanical Properties}

\subsubsection{Tensile Properties of 3D Printed Neat PLA and PLA/CB Nanocomposites}

In Figure 7, the tensile properties of 3D printed neat PLA, as well as PLA/CB nanocomposites with $0.1,0.5,1.0,2.5$ and $5.0 \mathrm{wt} . \% \mathrm{CB}$ filler loadings are presented. More specifically, Figure 7a illustrates the comparative and representative stress-strain curves for the different specimens. Figure $7 \mathrm{~b}$ shows the average tensile strength values, and Figure $7 \mathrm{c}$ the tensile modulus of elasticity average values, along with the corresponding standard deviations, all as a function of the filler loading. The CB imparts a negligible knock down effect at the lowest filler loading, while it yields enhanced tensile strength and modulus values for the rest i.e., 0.5, 1.0, 2.5 and $5.0 \mathrm{wt}$ \% f filler loadings. Accordingly, the PLA/CB with $0.5 \mathrm{wt} . \%$ $\mathrm{CB}$ exhibits the best performance and the highest reinforcing mechanism; namely $\sim 17.1 \%$ increase for the tensile strength and $\sim 14.1 \%$ increase for the tensile modulus, respectively.

By increasing further, the CB content in the PLA matrix, the CB efficiently enhances the tensile properties as compared to the reference neat PLA; however not as much prominently as the $0.5 \mathrm{wt} . \%$ loading. This could be explained by decreased filamentous interfacial strength between adjacent filaments in the bulk 3D printed sample and/or limited interdiffusion of the polymeric chains from one filament to another by the presence of $C B$ fillers, preventing an extended polymer chain mobility in the melt state at higher filler loadings. These findings also corroborate some small defects, inhomogeneities and interlayer voids observed by the SEM side surface images at the highest filler loading of $5.0 \mathrm{wt} . \%$. It can be deduced then that $0.5 \mathrm{wt} . \%$ of $\mathrm{CB}$ content is the mechanical percolation threshold of the 3D printed PLA/CB nanocomposites, endowing the optimum mechanical properties of the 3D printed specimens in quasi-static tension mode. 

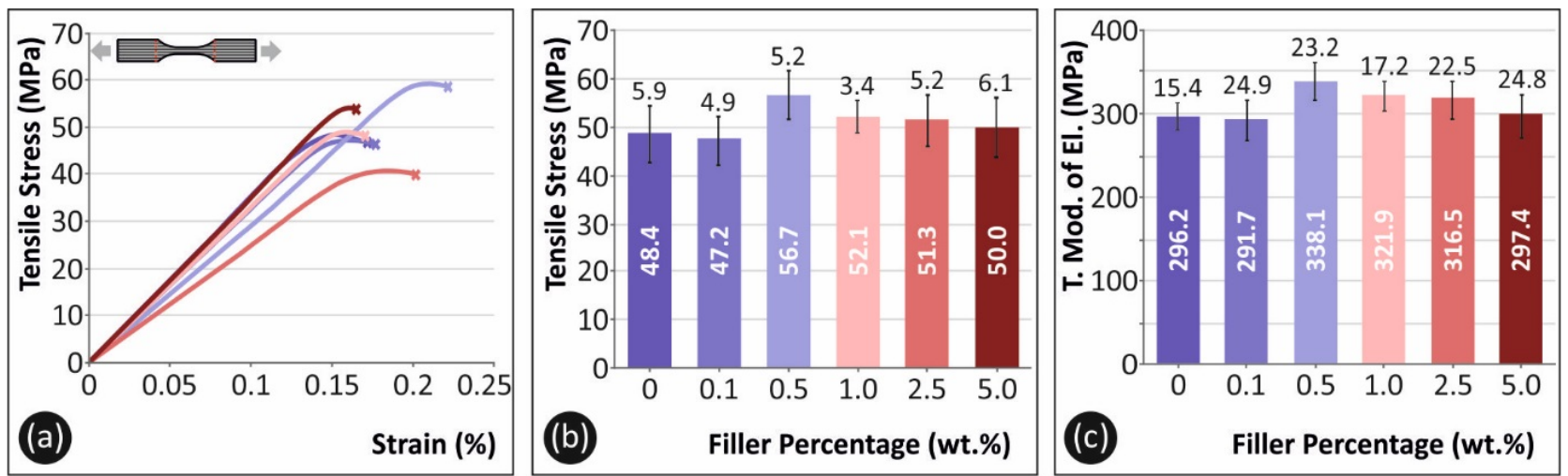

-PLA - PLA/CB 0.1\% - PLA/CB 0.5\% - PLA/CB 1.0\%

Figure 7. (a) Tensile stress (MPa) vs. strain (\%) representative curves for neat PLA and PLA/CB nanocomposites. (b) average tensile strength, and (c) average tensile modulus of elasticity values along with the calculated standard deviation values, both as a function of CB filler loading (wt.\%).

\subsubsection{SEM Fractography Microstructural Analysis}

Figure 8 depicts the fractography microstructural investigations of FFF 3D printed PLA nanocomposites after the tensile test experiments. The SEM images reveal both the specimens' fractured area characteristics and the specific fracture mechanism ("ductile" or "brittle" fracture mechanism), as well as the 3D printed sample's internal microstructure i.e., existence of voids, extent of the adjacent filament fusion, etc. Specifically, neat PLA (Figure 8a,b) compared to $0.5 \mathrm{wt} . \%$ (Figure 8c,d), $2.5 \mathrm{wt}$ \% (Figure 8e,f) and $5.0 \mathrm{wt} . \%$ (Figure 8g,h) CB filler loading SEM fractographic images are shown at two different magnifications, to elucidate the different underlying fracture mechanisms.

An excellent interdiffusion between the filaments, with no visible voids in the fractured surface can be seen only in the case of neat PLA. On the other hand, for all nanocomposites, some discontinuities and defects in the specimen's internal structure can be observed, with a general trend the internal structure to become worse with the increased filler loading. Furthermore, a relatively "brittle" fracture mechanism occurs for all the samples, seen from the low magnification images, since no filaments have been found to be pulled-out from the specimens' fractured surfaces.

At high magnification images, all samples exhibited almost similar characteristics with the surface microstructure PLA/CB nanocomposites exhibiting a slightly increased micro-roughness compared to the neat PLA. From the SEM fractographic analyses, some important findings and speculations could be deduced i.e., (i) no microaggregates could be observed in the nanocomposites' fractured surfaces even up to $5.0 \mathrm{wt} . \%$ filler loading, indirectly proving a sufficient $\mathrm{CB}$ nanodispersion (especially seen by the high magnification images), and (ii) a high-quality internal structure can be observed only for neat PLA, whereas PLA/CB nanocomposites exhibit some structural defects e.g., voids, etc., with the worst case at $5.0 \mathrm{wt} . \%$ filler loading. The fact that the neat PLA exhibited an excellent internal structure suggest that optimum set of printing parameters have been used in this study. 

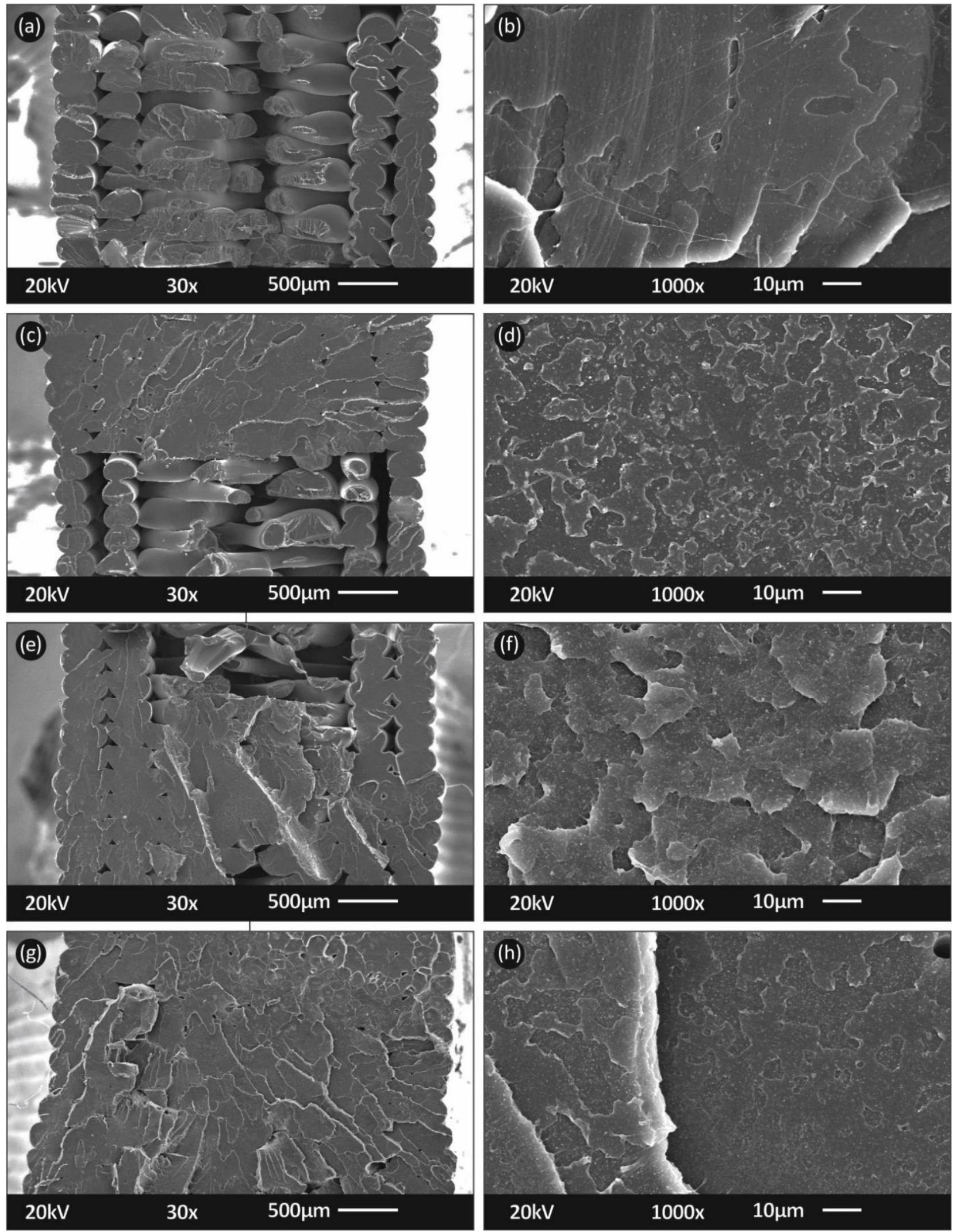

Figure 8. Fractured surface morphology of PLA/CB 3D printed nanocomposites after tensile tests: neat PLA (a,b) compared to 2.5 wt.\% (c,d), 5.0 wt.\% (e,f) and $10.0 \mathrm{wt} \%$ (g,h) CB filler loading, respectively. 


\subsubsection{Flexural Properties of 3D Printed Neat PLA and PLA/CB Nanocomposites}

Figure 9 summarizes the flexural properties of 3D printed neat PLA and PLA/CB nanocomposite specimens with 0.1, 0.5, 1.0, 2.5 and $5.0 \mathrm{wt}$ \% CB filler loadings. Figure $9 \mathrm{a}$ shows representative flexural stress-strain curves for the different tested samples. Figure $9 \mathrm{~b}$ illustrates the average flexural strength response, while Figure $9 c$, shows the average flexural modulus of elasticity of the 3D printed specimens. In all cases, the average values along with the calculated standard deviations are presented. Regarding the $\mathrm{CB}$ reinforcement in the flexion mode, interestingly enough, the 0.1 and $0.5 \mathrm{wt} . \%$ of CB impart a slight knock down effect compared to the reference neat PLA, both in the flexural strength and modulus. However, at 1.0, 2.5 and $5.0 \mathrm{wt} . \%$ the flexural properties are promising and a clear enhancement trend was observed, with the $2.5 \mathrm{wt} . \%$ PLA/CB exhibiting the best performance and the highest reinforcing mechanism; namely $~ 38.3 \%$ increase for the flexural strength and $\sim 31.2 \%$ increase for the flexural modulus, respectively. By increasing further, the CB content in the PLA matrix and reaching $5.0 \mathrm{wt} . \%, \mathrm{CB}$ efficiently enhances the flexural properties as compared to the reference neat PLA; however not as much prominently as the $2.5 \mathrm{wt} . \%$ loading. Overall, it can be presumed that at $2.5 \mathrm{wt} . \%$ of CB content, the mechanical percolation threshold is reached for the applied flexural strain field of the 3D printed PLA/CB nanocomposites.
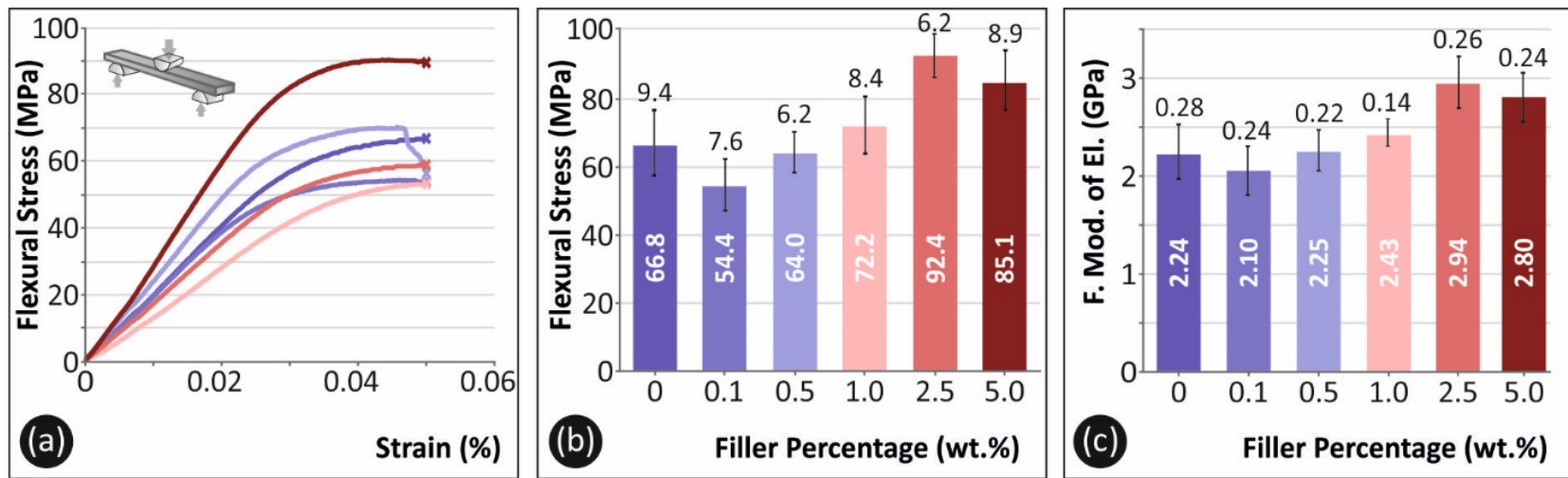

$-P L A \quad-P L A / C B$ 0.1\% $\quad-$ PLA/CB 0.5\% PLA/CB 1.0\% PLA/CB 2.5\%

Figure 9. (a) flexural stress (MPa) vs. strain (\%) representative curves for neat PLA and PLA/CB nanocomposites. (b) flexural strength and (c) flexural modulus of elasticity average values, along with the calculated standard deviation values, both as a function of the CB different filler loadings (wt.\%).

Although the highest calculated flexural strength was not developed on the same filler loading with the tensile tests, flexural tests results follow a similar trend to the tensile tests results. This verifies the reliability of the specimens' preparation process, since no significant differences or large deviations were observed between the tests results.

\subsubsection{Impact Properties and Micro-Hardness of 3D Printed Neat PLA and}

PLA/CB Nanocomposites

Figure 10a shows the average values of the Charpy's notched impact strength $\left(\mathrm{kJ} / \mathrm{m}^{2}\right)$ results for neat PLA and PLA/CB nanocomposites at different filler loadings. Figure 10b presents the respective micro-hardness (Vickers (HV)) response of the neat PLA and PLA/CB nanocomposites. The impact strength mean values are presented for the different 3D printed specimens in Figure 10a. As it can be observed, the impact strength is increasing by the presence of $\mathrm{CB}$ in the polymer matrix for all the filler loadings, while a very slight and negligible decrease could be observed for the $5.0 \mathrm{wt} . \%$ nanocomposite. Specifically, the highest impact strength improvement was found for the $1.0 \mathrm{wt} . \%$ nanocomposite $(\sim 24.6 \%)$. The slight decrease of the impact properties at $5.0 \mathrm{wt} . \%$ might be attributed to (i) some "critical" number of interlayer voids in the samples' side and internal structure at this CB nanoparticle loading, discussed in more detail in the SEM fractography analyses section, 
as well as (ii) a possible hampered polymer chain interdiffusion between the different layers and the adjacent filaments during melt deposition, due to the increase of the melt viscosity with the increased particle filler loading, accompanied with hindered polymer chain mobility.
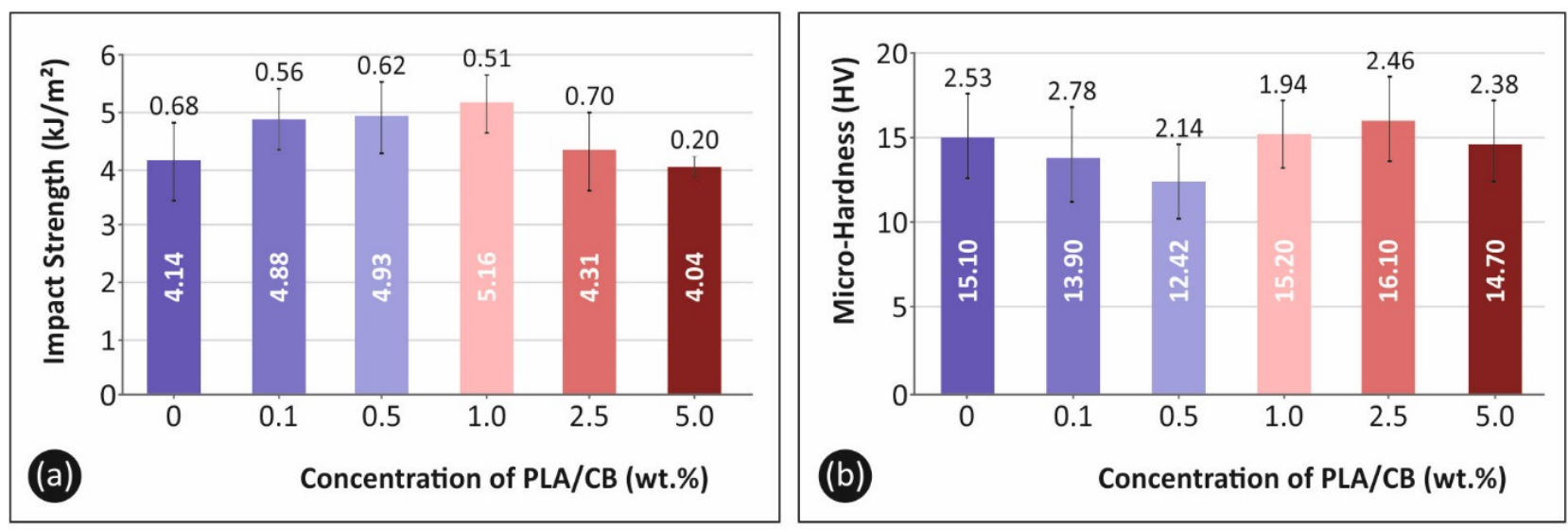

$-\mathrm{PLA} \quad-\mathrm{PLA} / \mathrm{CB} 0.1 \% \quad-\mathrm{PLA} / \mathrm{CB}$ 0.5\%

PLA/CB $1.0 \%$

- PLA/CB 2.5\%

- PLA/CB 5.0\%

Figure 10. (a) Charpy's notched impact strength test results $\left(\mathrm{kJ} / \mathrm{m}^{2}\right)$, and (b) micro-hardness (Vickers (HV)) performance of the neat PLA compared to the PLA/CB nanocomposites (both properties are shown as the calculated average values together with their corresponding standard deviation).

Regarding the micro-hardness (Figure 10b), the PLA/CB nanocomposites follow exactly the trend of the flexural properties with respect to the different filler loadings. Especially, the biggest improvement was observed for the $2.5 \mathrm{wt} . \%$ nanocomposite $(\sim 6.6 \%)$. The enhancement in the microhardness values is directly associated with the stiffening of the material induced by the CB nanoparticles in the PLA polymer matrix. Namely, micro-hardness slightly decreases for the 0.1 and $0.5 \mathrm{wt} . \%$ nanocomposites, while it slightly increases for the 1.0 and $2.5 \mathrm{wt} . \%$, with a slight decrease finally at the highest filled PLA at 5.0 wt. $\%$.

\subsection{Thermomechanical Analysis of 3D Printed Neat PLA and PLA/CB Nanocomposites}

Figure 11 illustrates the $3 \mathrm{D}$ printed specimens' thermomechanical response, i.e., the storage modulus ( $\left.\mathrm{E}^{\prime}, \mathrm{MPa}\right)$, loss modulus ( $\mathrm{E}^{\prime \prime}, \mathrm{MPa}$ ) and Tan $\delta$, as a function of temperature, derived from the DMA thermomechanical experiments. Storage moduli values at low temperatures $\left(\sim 30^{\circ} \mathrm{C}\right)$ coincide with the flexural moduli of the tested material, with the highest increase and reinforcing mechanism for the $2.5 \mathrm{wt} . \%$ nanocomposite. However, the $5.0 \mathrm{wt} . \%$ nanocomposite show also a promising thermomechanical reinforcement mechanism, which is an opposite behavior with regards to the flexural and the tensile properties. This interesting finding is attributed to the nature of the DMA set-up conditions and the samples' significantly lower dimensions, where statistically a plausible much smaller number of defects and discontinuities is present in the tested specimens affecting the bulk mechanical property. 

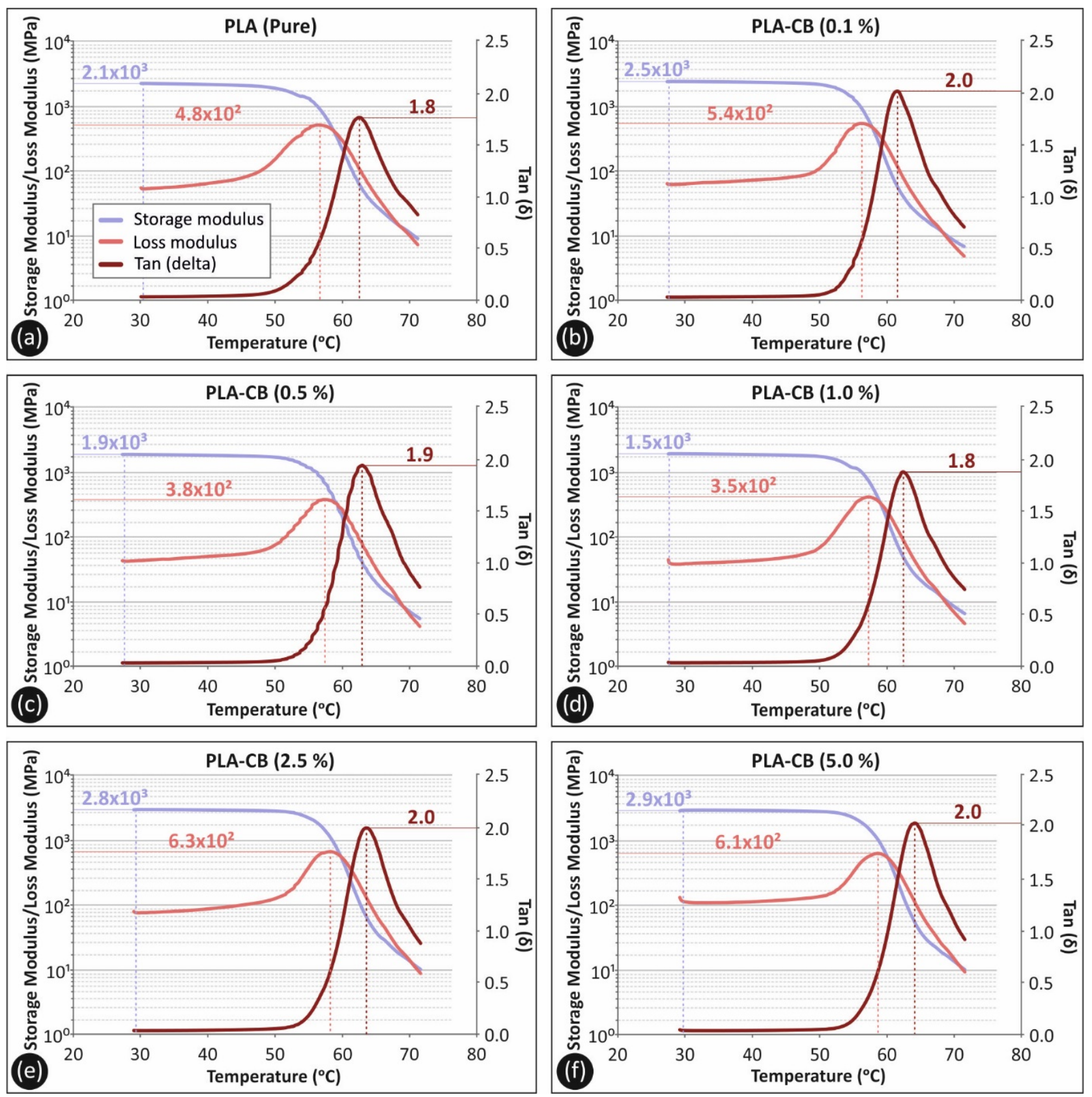

Figure 11. Storage modulus, loss modulus and Tan $\delta$ plots of the neat PLA and PLA/CB nanocomposites at different filler loadings: (a) pure PLA, (b) PLA/CB wt.\% 0.1, (c) PLA/CB wt.\% 0.5, (d) PLA/CB wt.\% 1.0, (e) PLA/CB wt.\% 2.5, (f) PLA/CB wt.\% 5.0.

The storage modulus reveals a decreasing tendency with the increase in temperature. The highest damping is recorded up to $0.1 \mathrm{wt} . \%$ doped samples, and marginally decreased for the $0.5,1.0,2.5$ and $5.0 \mathrm{wt} . \%$ nanocomposites. The Tan $\delta$ values for all samples exhibit an increasing trend up to the temperature range of $62{ }^{\circ} \mathrm{C}$, indicating that up to this temperature range the mechanical energy is stored elastically in the polymeric macromolecular chains; however, with a decreased ability as the temperature increases, manifested by the decreased storage moduli values. On the other hand, $>60-65{ }^{\circ} \mathrm{C}$ the Tan $\delta$ values show a drop, revealing the softening of the material before fusion, and the transition to the viscoelastic regime. It is worth mentioning that the increase in the storage moduli for all the nanocomposite samples found by the DMA experiments is associated with the stiffening of the polymeric chains in the presence of CB nanofillers. The highest storage 
modulus value was observed for the $2.5 \mathrm{wt} . \%$, which was also almost identical with the $5.0 \mathrm{wt} . \%$ nanocomposite, exhibiting an increase of approximately $40 \%$ when compared to the neat PLA.

\subsection{Antibacterial Performance of the PLA/CB 3D Printed Nanocomposites}

Figure 12 demonstrates the antibacterial results after the $24 \mathrm{~h}$ bacteria culture for the highest concentration PLA/CB nanocomposite studied in this research work (5.0 wt.\%). A mild clearly visible antibacterial activity was observed, while non-measurable antimicrobial activity has been found for the PLA/CB at lower filler loadings and no antibacterial performance was found for the neat PLA, with the process followed. The developed Inhibition Zone (IZ) was almost the same for both Staphylococcus aureus and Escherichia coli bacteria. The antibacterial results presented in Figure 12 show that the addition of CB in the PLA matrix can introduce antibacterial properties to the polymer, at least for the two bacteria tested in this study. It should be mentioned that the agar well diffusion method employed in this work exposes the specimens in a severe bacteria environment and it is suitable mainly as a screening process, providing information regarding the potential of the material's antibacterial performance. Still, this is an important finding, considering that the developed nanocomposites were 3D printed, making it possible to build on-demand parts with FFF 3D printing process exhibiting antibacterial properties.
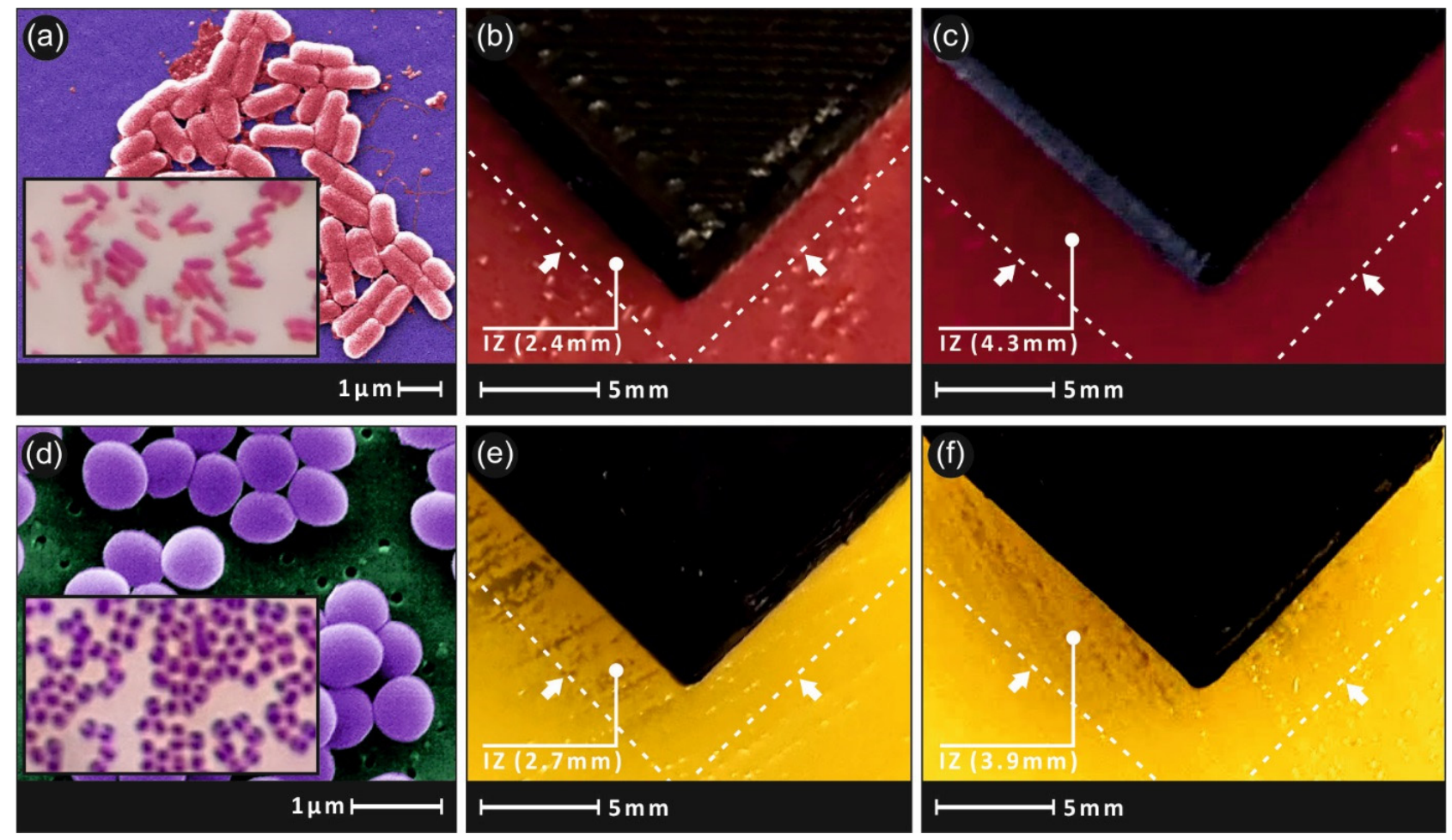

Figure 12. Agar well diffusion method pictures after $24 \mathrm{~h}$ culture showing the Inhibition Zone (IZ) of (a) Escherichia coli bacteria against PLA/CB (5.0 wt.\%) (b-d) and Staphylococcus aureus against PLA/CB (5.0 wt.\%) (e,f).

\section{Conclusions}

PLA/CB nanocomposites at 0.5, 1.0, 2.5 and $5.0 \mathrm{wt}$ \% filler loadings were produced by melt mixing followed by a filament extrusion process, to produce the feedstock material utilized for the FFF 3D printing consecutive manufacturing of PLA/CB. All filament extrusion and 3D printing parameters have been initially optimized, yielding high quality specimens and an efficient 3D printing process. Raman spectroscopy revealed the graphitic nature of $\mathrm{CB}$, as well as the corresponding PLA matrix characteristic fingerprints, and the PLA/CB nanocomposites (at $2.5 \mathrm{wt}$.\% filler loading) peaks, due to the constituent matrix and dispersed filler phases. TGA analysis proved that the temperatures employed 
during the extrusion and the 3D printing process are appropriate for the materials prepared and tested in this work and the overall thermal stability of the samples does not seem to be impacted. The PLA/CB 3D printed nanocomposites have been fully investigated for their static and thermomechanical properties; while they have been fully analyzed in terms of their electrically conductivity and demonstrated as (i) Joule heater devices and (ii) conductive circuitry resistor multi-functional nanocomposites.

The electrical percolation threshold was studied, with the nanocomposites exhibiting an electrically conductive behavior from the $2.5 \mathrm{wt} . \%$ loading and increasing by increasing the filler loading up to $5.0 \mathrm{wt} . \%$. The nanocomposites showed an anisotropic electrically conductive behavior in the "through" thickness and "cross-layer" 3D printing specimens" measurement directions. The highest electrical conductivity $(\sigma)$ was obtained at the "crosslayer" direction for the PLA/CB $(5.0 \mathrm{wt} . \%)$ nanocomposite $(\sim 0.9 \mathrm{~S} / \mathrm{m})$, while it exhibited an electrothermal "Joule-heating" function with its surface temperature raising at $\sim 35^{\circ} \mathrm{C}$ within $30 \mathrm{sec}$. upon being to a voltage bias $\left(\mathrm{V}_{\text {bias }}\right)$ of $15 \mathrm{~V}$. It is worth to be mentioned that PLA/CB (10.0 wt.\%) electrically conductive filaments were also produced; however, they were not processable towards a continuous FFF 3D printing process, hence they are not included this work.

Detailed mechanical investigations i.e., tensile, flexural, impact, microhardness and Dynamic Mechanical Analysis (DMA) tests revealed the structure-property relationship of the fabricated 3D printed nanocomposite materials, as well as elucidating the mechanical reinforcement mechanisms under static and dynamic mechanical loadings for the PLA/CB nanocomposites. Scanning Electron Microscopy (SEM) fractographic analyses revealed the underlying fracture mechanisms of the tensile tested specimens. Additionally, the agar wellknown diffusion method revealed a mild antibacterial performance of the nanocomposite at the highest filler loading of $5.0 \mathrm{wt} . \%$ prepared in this work. The overall results especially related to the mechanical performance of the 3D printed nanocomposites manufactured and tested in this study are summarized in Figure 13. As it is shown, 0.5 wt.\% PLA/CB $\mathrm{CPC}$ had the highest tensile strength enhancement, although higher loadings had similar tensile strength results. In the remaining mechanical properties, the $2.5 \mathrm{wt}$ \% PLA/CB CPC had the most enhanced performance, making it overall the best filler loading, regarding the mechanical response of the nanocomposite studied in this work.

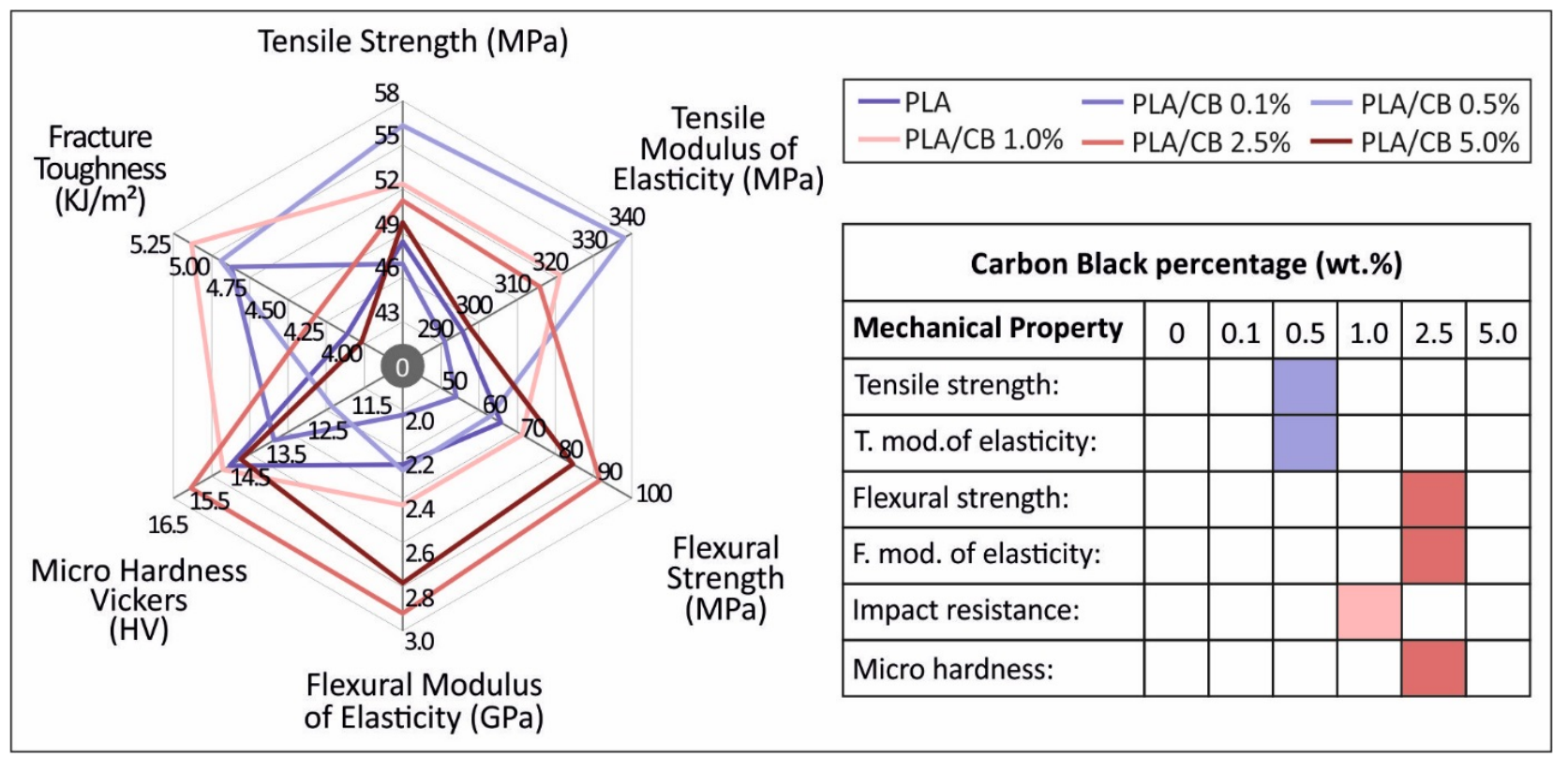

Figure 13. Overall results on the mechanical properties and performance of neat PLA compared to PLA/CB 3D printed nanocomposites. 
It could be envisaged that the proposed multi-functional 3D printed PLA/CB CPC manufactured nanocomposites with enhanced mechanical and electrical properties, Jouleheating capability and antibacterial properties could create the seeds for future 3D shaped multifunctional objects where complex 3D architectures and customizability are required; i.e., low-power demanding applications such as wearables, flexible conductors, resistors for circuitry, IoT sensors and personalized biomedical components (wearable strain sensors, antimicrobial splints, etc.).

Author Contributions: Conceptualization, N.V. and L.T.; methodology, M.P.; software, N.M.; validation, L.T., E.V. and S.G.; formal analysis, N.M., P.E.F.-G. and M.P.; investigation, N.M. and E.V.; resources, N.V., data curation, P.E.F.-G. and N.M.; writing-original draft preparation, E.V., L.T. and S.G.; writing-review and editing, E.V., M.P. and S.G.; visualization, P.E.F.-G. and N.V.; supervision, N.V.; project administration, M.P.; funding acquisition, N.V. The manuscript was written through contributions of all authors. All authors have read and agreed to the published version of the manuscript.

Funding: This research received no external funding.

Institutional Review Board Statement: Not applicable.

Informed Consent Statement: Not applicable.

Data Availability Statement: The data presented in this study are available on request from the corresponding author.

Acknowledgments: Authors would like to thank Aleka Manousaki from the University of Crete for taking the SEM images presented in this work.

Conflicts of Interest: The authors declare no conflict of interest.

\section{References}

1. Tzounis, L.; Petousis, M.; Grammatikos, S.; Vidakis, N. 3D Printed Thermoelectric Polyurethane/Multiwalled Carbon Nanotube Nanocomposites: A Novel Approach towards the Fabrication of Flexible and Stretchable Organic Thermoelectrics. Materials 2020, 13, 2879. [CrossRef] [PubMed]

2. Kapnopoulos, C.; Mekeridis, E.D.; Tzounis, L.; Polyzoidis, C.; Zachariadis, A.; Tsimikli, S.; Gravalidis, C.; Laskarakis, A.; Vouroutzis, N.; Logothetidis, S. Fully gravure printed organic photovoltaic modules: A straightforward process with a high potential for large scale production. Sol. Energy Mater. Sol. Cells 2016, 144, 724-731. [CrossRef]

3. Tzounis, L.; Bangeas, P.I.; Exadaktylos, A.; Petousis, M.; Vidakis, N. Three-Dimensional Printed Polylactic Acid (PLA) Surgical Retractors with Sonochemically Immobilized Silver Nanoparticles: The Next Generation of Low-Cost Antimicrobial Surgery Equipment. Nanomaterials 2020, 10, 985. [CrossRef]

4. Zhang, J.; Wang, J.; Dong, S.; Yu, X.; Han, B. A review of the current progress and application of 3D printed concrete. Compos. Part A Appl. Sci. Manuf. 2019, 125, 105533. [CrossRef]

5. Gnanasekaran, K.; Heijmans, T.; van Bennekom, S.; Woldhuis, H.; Wijnia, S.; de With, G.; Friedrich, H. 3D printing of CNT- and graphene-based conductive polymer nanocomposites by fused deposition modeling. Appl. Mater. Today 2017, 9, 21-28. [CrossRef]

6. Takagishi, K.; Umezu, S. Development of the Improving Process for the 3D Printed Structure. Sci. Rep. 2017, 7, 39852. [CrossRef]

7. Velu, R.; Raspall, F.; Singamneni, S. Chapter 8-3D printing technologies and composite materials for structural applications. In Green Composites for Automotive Applications; Koronis, G., Silva, A., Eds.; Woodhead Publishing: Sawston, UK, 2019 ; pp. $171-196$.

8. Tian, X.; Liu, T.; Yang, C.; Wang, Q.; Li, D. Interface and performance of 3D printed continuous carbon fiber reinforced PLA composites. Compos. Part A Appl. Sci. Manuf. 2016, 88, 198-205. [CrossRef]

9. Vidakis, N.; Petousis, M.; Tzounis, L.; Maniadi, A.; Velidakis, E.; Mountakis, N.; Kechagias, J.D. Sustainable Additive Manufacturing: Mechanical Response of Polyamide 12 over Multiple Recycling Processes. Materials 2021, 14, 466. [CrossRef] [PubMed]

10. Ngo, T.D.; Kashani, A.; Imbalzano, G.; Nguyen, K.T.Q.; Hui, D. Additive manufacturing (3D printing): A review of materials, methods, applications and challenges. Compos. Part B Eng. 2018, 143, 172-196. [CrossRef]

11. Savvakis, K.; Petousis, M.; Vairis, A.; Vidakis, N.; Bikmeyev, A.T. Experimental Determination of the Tensile Strength of Fused Deposition Modeling Parts. In Proceedings of the ASME 2014 International Mechanical Engineering Congress and Exposition, Montreal, QC, Canada, 14-20 November 2014.

12. Vidakis, N.; Petousis, M.; Vairis, A.; Savvakis, K.; Maniadi, A. On the compressive behavior of an FDM Steward Platform part. J. Comput. Des. Eng. 2017, 4, 339-346. [CrossRef]

13. Vidakis, N.; Petousis, M.; Vairis, A.; Savvakis, K.; Maniadi, A. A parametric determination of bending and Charpy's impact strength of ABS and ABS-plus fused deposition modeling specimens. Prog. Addit. Manuf. 2019, 4, 323-330. [CrossRef] 
14. Tan, L.J.; Zhu, W.; Zhou, K. Recent Progress on Polymer Materials for Additive Manufacturing. Adv. Funct. Mater. 2020, 30, 2003062. [CrossRef]

15. Vidakis, N.; Petousis, M.; Savvakis, K.; Maniadi, A.; Koudoumas, E. A comprehensive investigation of the mechanical behavior and the dielectrics of pure polylactic acid (PLA) and PLA with graphene (GnP) in fused deposition modeling (FDM). Int. J. Plast. Technol. 2019, 23, 195-206. [CrossRef]

16. Chacón, J.M.; Caminero, M.A.; García-Plaza, E.; Núñez, P.J. Additive manufacturing of PLA structures using fused deposition modelling: Effect of process parameters on mechanical properties and their optimal selection. Mater. Des. 2017, 124, 143-157. [CrossRef]

17. Vidakis, N.; Maniadi, A.; Petousis, M.; Vamvakaki, M.; Kenanakis, G.; Koudoumas, E. Mechanical and Electrical Properties Investigation of 3D-Printed Acrylonitrile-Butadiene-Styrene Graphene and Carbon Nanocomposites. J. Mater. Eng. Perform. 2020, 29, 1909-1918. [CrossRef]

18. Taylor, A.P.; Cuervo, C.V.; Arnold, D.P.; Velásquez-García, L.F. Fully 3D-Printed, Monolithic, Mini Magnetic Actuators for Low-Cost, Compact Systems. J. Microelectromech. Syst. 2019, 28, 481-493. [CrossRef]

19. Hohimer, C.J.; Petrossian, G.; Ameli, A.; Mo, C.; Pötschke, P. 3D printed conductive thermoplastic polyurethane/carbon nanotube composites for capacitive and piezoresistive sensing in soft pneumatic actuators. Addit. Manuf. 2020, $34,101281$.

20. Le, T.; Song, B.; Liu, Q.; Bahr, R.A.; Moscato, S.; Wong, C.; Tentzeris, M.M. A novel strain sensor based on 3D printing technology and 3D antenna design. In Proceedings of the 2015 IEEE 65th Electronic Components and Technology Conference (ECTC), San Diego, CA, USA, 26-29 May 2015; pp. 981-986.

21. Vidakis, N.; Petousis, M.; Maniadi, A.; Koudoumas, E.; Liebscher, M.; Tzounis, L. Mechanical Properties of 3D-Printed Acrylonitrile-Butadiene-Styrene $\mathrm{TiO}_{2}$ and ATO Nanocomposites. Polymers 2020, 12, 1589. [CrossRef]

22. Vidakis, N.; Petousis, M.; Velidakis, E.; Mountakis, N.; Tzounis, L.; Liebscher, M.; Grammatikos, S.A. Enhanced Mechanical, Thermal and Antimicrobial Properties of Additively Manufactured Polylactic Acid with Optimized Nano Silica Content. Nanomaterials 2021, 11, 1012. [CrossRef] [PubMed]

23. Thaler, D.; Aliheidari, N.; Ameli, A. Mechanical, electrical, and piezoresistivity behaviors of additively manufactured acrylonitrile butadiene styrene/carbon nanotube nanocomposites. Smart Mater. Struct. 2019, 28, 084004. [CrossRef]

24. Vidakis, N.; Petousis, M.; Velidakis, E.; Liebscher, M.; Tzounis, L. Three-Dimensional Printed Antimicrobial Objects of Polylactic Acid (PLA)-Silver Nanoparticle Nanocomposite Filaments Produced by an In-Situ Reduction Reactive Melt Mixing Process. Biomimetics 2020, 5, 42. [CrossRef] [PubMed]

25. Tzounis, L.; Liebscher, M.; Fuge, R.; Leonhardt, A.; Mechtcherine, V. P- and n-type thermoelectric cement composites with CVD grown p- and n-doped carbon nanotubes: Demonstration of a structural thermoelectric generator. Energy Build. 2019, 191, 151-163. [CrossRef]

26. Tzounis, L. Chapter 9-Synthesis and Processing of Thermoelectric Nanomaterials, Nanocomposites, and Devices. In Nanomaterials Synthesis; Pottathara, Y.B., Thomas, S., Kalarikkal, N., Grohens, Y., Kokol, V., Eds.; Elsevier: Amsterdam, The Netherlands, 2019; pp. 295-336.

27. Foteinidis, G.; Tsirka, K.; Tzounis, L.; Baltzis, D.; Paipetis, A.S. The Role of Synergies of MWCNTs and Carbon Black in the Enhancement of the Electrical and Mechanical Response of Modified Epoxy Resins. Appl. Sci. 2019, 9, 3757. [CrossRef]

28. Papageorgiou, D.G.; Tzounis, L.; Papageorgiou, G.Z.; Bikiaris, D.N.; Chrissafis, K. $\beta$-nucleated propylene-ethylene random copolymer filled with multi-walled carbon nanotubes: Mechanical, thermal and rheological properties. Polymer 2014, 55, 3758-3769. [CrossRef]

29. Tzounis, L.; Pegel, S.; Zafeiropoulos, N.E.; Avgeropoulos, A.; Paipetis, A.S.; Stamm, M. Shear alignment of a poly(styrenebutadiene-styrene) triblock copolymer/MWCNT nanocomposite. Polymer 2017, 131, 1-9. [CrossRef]

30. Zhou, B.; Luo, W.; Yang, J.; Duan, X.; Wen, Y.; Zhou, H.; Chen, R.; Shan, B. Thermal conductivity of aligned CNT/polymer composites using mesoscopic simulation. Compos. Part A Appl. Sci. Manuf. 2016, 90, 410-416. [CrossRef]

31. Tzounis, L.; Zappalorto, M.; Panozzo, F.; Tsirka, K.; Maragoni, L.; Paipetis, A.S.; Quaresimin, M. Highly conductive ultra-sensitive SWCNT-coated glass fiber reinforcements for laminate composites structural health monitoring. Compos. Part B Eng. 2019, 169, 37-44. [CrossRef]

32. Han, T.; Kundu, S.; Nag, A.; Xu, Y. 3D Printed Sensors for Biomedical Applications: A Review. Sensors 2019, 19, 1706. [CrossRef]

33. Christ, J.F.; Aliheidari, N.; Ameli, A.; Pötschke, P. 3D printed highly elastic strain sensors of multiwalled carbon nanotube/thermoplastic polyurethane nanocomposites. Mater. Des. 2017, 131, 394-401. [CrossRef]

34. Haghiashtiani, G.; Habtour, E.; Park, S.-H.; Gardea, F.; McAlpine, M.C. 3D printed electrically-driven soft actuators. Extrem. Mech. Lett. 2018, 21, 1-8. [CrossRef]

35. Jaksic, N.I.; Desai, P.D. Characterization of resistors created by fused filament fabrication using electrically-conductive filament. Procedia Manuf. 2018, 17, 37-44. [CrossRef]

36. Liu, S.; Li, Y.; Shen, Y.; Lu, Y. Mechanical performance of carbon fiber/epoxy composites cured by self-resistance electric heating method. Int. J. Adv. Manuf. Technol. 2019, 103, 3479-3493. [CrossRef]

37. Ibrahim, Y.; Kempers, R.; Amirfazli, A. 3D printed electro-thermal anti- or de-icing system for composite panels. Cold Reg. Sci. Technol. 2019, 166, 102844. [CrossRef]

38. Aumnate, C.; Potiyaraj, P.; Saengow, C.; Giacomin, A.J. Reinforcing polypropylene with graphene-polylactic acid microcapsules for fused-filament fabrication. Mater. Des. 2021, 198, 109329. [CrossRef] 
39. Shmueli, Y.; Lin, Y.-C.; Zuo, X.; Guo, Y.; Lee, S.; Freychet, G.; Zhernenkov, M.; Kim, T.; Tannenbaum, R.; Marom, G.; et al. In-situ $\mathrm{X}$-ray scattering study of isotactic polypropylene/graphene nanocomposites under shear during fused deposition modeling 3D printing. Compos. Sci. Technol. 2020, 196, 108227. [CrossRef]

40. Lei, L.; Yao, Z.; Zhou, J.; Wei, B.; Fan, H. 3D printing of carbon black/polypropylene composites with excellent microwave absorption performance. Compos. Sci. Technol. 2020, 200, 108479. [CrossRef]

41. Kwok, S.W.; Goh, K.H.H.; Tan, Z.D.; Tan, S.T.M.; Tjiu, W.W.; Soh, J.Y.; Ng, Z.J.G.; Chan, Y.Z.; Hui, H.K.; Goh, K.E.J. Electrically conductive filament for 3D-printed circuits and sensors. Appl. Mater. Today 2017, 9, 167-175. [CrossRef]

42. Vidakis, N.; Petousis, M.; Tzounis, L.; Velidakis, E.; Mountakis, N.; Grammatikos, S.A. Polyamide 12/Multiwalled Carbon Nanotube and Carbon Black Nanocomposites Manufactured by 3D Printing Fused Filament Fabrication: A Comparison of the Electrical, Thermoelectric, and Mechanical Properties. C 2021, 7, 38.

43. Jing, J.; Chen, Y.; Shi, S.; Yang, L.; Lambin, P. Facile and scalable fabrication of highly thermal conductive polyethylene/graphene nanocomposites by combining solid-state shear milling and FDM 3D-printing aligning methods. Chem. Eng. J. 2020, 402 , 126218. [CrossRef]

44. Tan, J.C.; Low, H.Y. Multi-materials fused filament printing with embedded highly conductive suspended structures for compressive sensing. Addit. Manuf. 2020, 36, 101551. [CrossRef]

45. Petroni, J.M.; Neves, M.M.; de Moraes, N.C.; Bezerra da Silva, R.A.; Ferreira, V.S.; Lucca, B.G. Development of highly sensitive electrochemical sensor using new graphite/acrylonitrile butadiene styrene conductive composite and 3D printing-based alternative fabrication protocol. Anal. Chim. Acta 2021, 1167, 338566. [CrossRef]

46. João, A.F.; Castro, S.V.F.; Cardoso, R.M.; Gamela, R.R.; Rocha, D.P.; Richter, E.M.; Muñoz, R.A.A. 3D printing pen using conductive filaments to fabricate affordable electrochemical sensors for trace metal monitoring. J. Electroanal. Chem. 2020, 876, 114701. [CrossRef]

47. Flowers, P.F.; Reyes, C.; Ye, S.; Kim, M.J.; Wiley, B.J. 3D printing electronic components and circuits with conductive thermoplastic filament. Addit. Manuf. 2017, 18, 156-163. [CrossRef]

48. Abdalla, A.; Hamzah, H.H.; Keattch, O.; Covill, D.; Patel, B.A. Augmentation of conductive pathways in carbon black/PLA 3D-printed electrodes achieved through varying printing parameters. Electrochim. Acta. 2020, 354, 136618. [CrossRef]

49. Balouiri, M.; Sadiki, M.; Ibnsouda, S.K. Methods for in vitro evaluating antimicrobial activity: A review. J. Pharm. Anal. 2016, 6, 71-79. [CrossRef] [PubMed]

50. Li, Z.; Zabihi, O.; Wang, J.; Li, Q.; Wang, J.; Lei, W.; Naebe, M. Hydrophilic PAN based carbon nanofibres with improved graphitic structure and enhanced mechanical performance using ethylenediamine functionalized graphene. RSC Adv. 2017, 7, 2621-2628. [CrossRef]

51. Felisberto, M.; Tzounis, L.; Sacco, L.; Stamm, M.; Candal, R.; Rubiolo, G.H.; Goyanes, S. Carbon nanotubes grown on carbon fiber yarns by a low temperature CVD method: A significant enhancement of the interfacial adhesion between carbon fiber/epoxy matrix hierarchical composites. Compos. Commun. 2017, 3, 33-37. [CrossRef]

52. Tzounis, L.; Petousis, M.; Liebscher, M.; Grammatikos, S.; Vidakis, N. Three-Dimensional (3D) Conductive Network of CNTModified Short Jute Fiber-Reinforced Natural Rubber: Hierarchical CNT-Enabled Thermoelectric and Electrically Conductive Composite Interfaces. Materials 2020, 13, 2668. [CrossRef] [PubMed]

53. Liebscher, M.; Tzounis, L.; Junger, D.; Dinh, T.T.; Mechtcherine, V. Electrical Joule heating of cementitious nanocomposites filled with multi-walled carbon nanotubes: Role of filler concentration, water content, and cement age. Smart Mater. Struct. 2020, 29, 125019. [CrossRef] 